University of South Florida

DIGITAL COMMONS

Digital Commons @ University of

@ UNIVERSITY OF SOUTH FLORIDA

South Florida

$1-1-2015$

\title{
2015 Work Plan USF Tampa
}

USF

Follow this and additional works at: https://digitalcommons.usf.edu/usf_accountability_reports

\section{Scholar Commons Citation}

USF, "2015 Work Plan USF Tampa" (2015). USF Accountability Reports. 56.

https://digitalcommons.usf.edu/usf_accountability_reports/56

This Article is brought to you for free and open access by the USF Archives at Digital Commons @ University of South Florida. It has been accepted for inclusion in USF Accountability Reports by an authorized administrator of Digital Commons @ University of South Florida. For more information, please contact digitalcommons@usf.edu. 


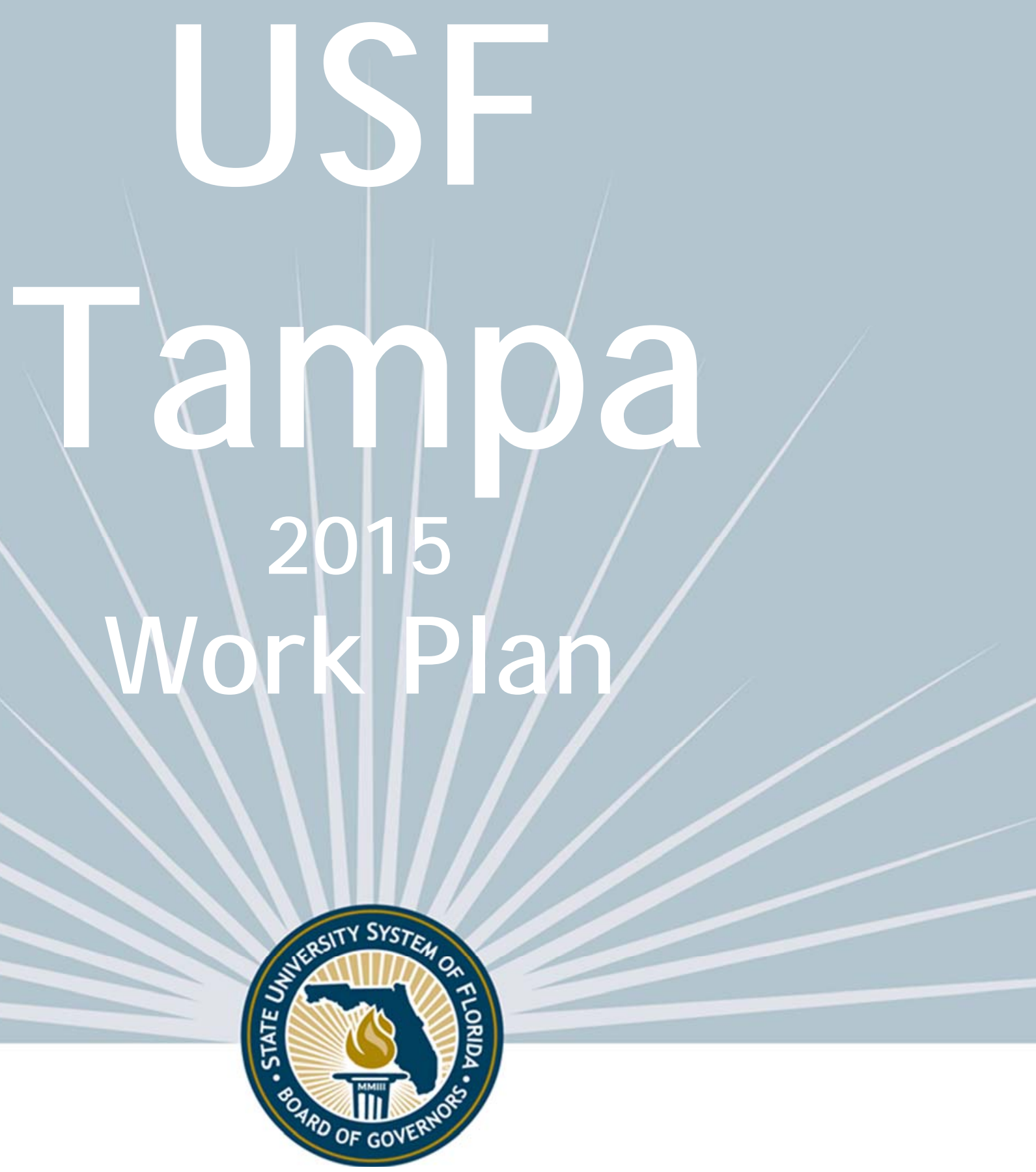

University of South Florida - Tampa

University Work Plan Presentation

for Board of Governors June 2015 Meeting

STATE UNIVERSITY SYSTEM of FLORIDA $\mid$ Board of Governors 


\section{INTRODUCTION}

The State University System of Florida has developed three tools that aid in guiding the System's future.

1) The Board of Governors' 2025 System Strategic Plan is driven by goals and associated metrics that stake out where the System is headed;

2) The Board's Annual Accountability Report provides yearly tracking for how the System is progressing toward its goals;

3) Institutional Work Plans connect the two and create an opportunity for greater dialogue relative to how each institution contributes to the System's overall vision.

These three documents assist the Board with strategic planning and with setting short-, mid- and long-term goals. They also enhance the System's commitment to accountability and driving improvements in three primary areas of focus: 1) academic quality, 2) operational efficiency, and 3) return on investment.

The Board will use these documents to help advocate for all System institutions and foster even greater coordination with the institutions and their Boards of Trustees.

Once a Work Plan is approved by each institution's respective Boards of Trustees, the Board of Governors will review and consider the plan for potential acceptance of 2015-16 components. Longer-term components will inform future agendas of the Board's Strategic Planning Committee. The Board's acceptance of a work plan does not constitute approval of any particular component, nor does it supersede any necessary approval processes that may be required for each component. 


\section{TABLE OF CONTENTS}

\section{STRATEGY}

a. Mission Statement

b. Vision Statement

c. Statement of Strategy

d. Strengths and Opportunities

e. Key Initiatives \& Investments

\section{PERFORMANCE BASED FUNDING METRICS}

\section{PREEMINENT RESEARCH UNIVERSITY METRICS}

\section{OTHER KEY PERFORMANCE INDICATORS}

a. Goals Common to All Universities

b. Goals Specific to Research Universities

c. Institution Specific Goals

\section{OPERATIONS}

a. Fiscal Information

b. Enrollment Planning

c. Academic Program Coordination

\section{DEFINITIONS}




\section{MISSION STATEMENT (What is your purpose?)}

The University of South Florida's mission is to deliver competitive undergraduate, graduate, and professional programs, to generate knowledge, foster intellectual development, and ensure student success in a global environment.

\section{VISION STATEMENT (What do you aspire to?)}

The University of South Florida is a global research university dedicated to student success and positioned for membership in the Association of American Universities (AAU).

As Florida's leading metropolitan research university, USF is dedicated to:

- Student access, learning, and success through a vibrant, interdisciplinary, and learner-centered research environment, incorporating a global curriculum.

- Research and scientific discovery to strengthen the economy, promote civic engagement, culture, and the arts, and design and build sustainable communities through the generation, dissemination and translation of new knowledge across all academic and health-related disciplines.

- Partnerships to build significant locally and globally integrated university-community collaborations through sound scholarly and artistic activities and technological innovation.

- A sustainable economic base to support USF's continued academic advancement.

\section{STATEMENT OF STRATEGY (How will you get there?)}

Given your mission, vision, strengths and available resources, provide a brief description of your market and your strategy for addressing and leading it.

USF is a Carnegie classified very high research university, attracting students and faculty of the highest caliber. As USF moves forward in positioning itself for AAU eligibility, it maintains its sharply focused commitment to student success, along with embracing entrepreneurship and innovation at all levels.

The foundation of USF's strategy is accountability, guided by the Board of Governors' Strategic Plan, USF's Strategic Plan and the Board of Governors' Performance-Funding model. By benchmarking progress to our current and aspirational peer institutions, a clear roadmap is created which guides USF toward further excellence and, ultimately, AAU membership.

As part of a regular review process, the USF Board of Trustees and USF leadership use a detailed crosswalk to track key metrics for performance-based funding, preeminence and AAU membership, such as graduation rates, retention rates, research expenditures and faculty awards. The reviews guide decision-making for resource allocation to ensure maximum impact. For example, this past year a Freshman Retention Advocate was added to the team of specialized trained academic advisors, who collectively monitor issues affecting persistence and retention, while developing approaches to move students towards graduation. Endeavors like this one, along with continued policy review and targeted hires, have again resulted in an improvement in the graduation rate and in the number of students graduating without excess hours, among other successes.

USF cultivates strong collaborations with the Tampa Bay community and beyond, including some of Florida's largest businesses, to maximize collaborative opportunities, including student internships. Seeking to educate globally-competitive graduates, USF encourages education abroad, recruits talented international students, and incorporates a global focus into the curriculum across the university. 


\section{STRENGTHS AND OPPORTUNITIES (within 3 years)}

\section{What are your core capabilities, opportunities and challenges for improvement?}

One of USF's key strengths is its agility. We respond quickly to challenges, continually looking for ways to improve, and readily accept new opportunities for growth. This attitude helps us quickly direct focus to those areas that are most important to our students and the Board of Governors. This is reflected in our steady gains in graduation and retention rates, the culture of innovation that has made USF one of the most productive research universities in Florida, and our ever-expanding network of partners in the community.

Guided by that spirit, we have readily embraced the Board of Governors' performance-based funding model, strengthening our culture of transparency, accountability and commitment to student success. Thanks to the performance-funding investments we have received as a top performer the past few years, we have been able to enhance the quality of our education and advising services while continuing to keep costs down for students. Our challenge will continue to be maintaining that momentum as we seek further budget efficiencies. Reduced federal research funds also continue to be a challenge for all research universities.

\section{KEY INITIATIVES \& INVESTMENTS (within 3 years)}

Describe your top three key initiatives for the next three years that will drive improvement in Academic Quality, Operational Efficiency, and Return on Investment.

\section{Graduate well-educated global citizens through a continued commitment to student success:}

USF's No. 1 priority remains to deliver high quality, relevant and globally informed academic programs to prepare graduates for leadership roles and workforce opportunities both locally and across the world. This commitment is guided by our efforts to further improve student success through personalized advising, research opportunities at every level, and career-readiness programs.

USF has committed to its global focus through its Quality Enhancement Plan for SACS reaccreditation this year. USF's QEP is dedicated to developing "global citizens." USF will acomplish this by infusing curriculum across campus with global, cross-cultural perspectives and ensuring every student has an opportunity for a global experience - whether through a technology-enabled global classroom, a study abroad experience, or meaningful interactions with international students and cultures.

\section{Produce high-impact research and innovation that will change lives for the better, improve health, and foster positive societal change:}

USF has escalated its efforts to recruit and retain research-productive new faculty; intensified its encouragement of undergraduate research that will contribute to the overall positive education experience and enhance student success; supported interdisciplinary initiatives that aim to solve critical problems; provided training to increase external funding; and continued to promote partnerships across the university and within the broader community.

USF is again working to attain status as an Innovation and Prosperity University from the Association of Public and Land Grant Universities - a process that has brought together USF leadership, researchers, government stakeholders and local business leaders. The application process alone has created a culture of collaboration for translating research into meaningful applications that will have an impact on the world. USF looks forward to continued progress in this process. 
3. Create new partnerships, seek new efficiencies, and cultivate opportunities that will maintain USF's position as a highly effective economic engine for Florida:

USF's partnerships strengthen the Tampa Bay region and Florida as part of the global landscape. USF also continually works to maximize efficiencies and use its resources in the most prudent ways possible-especially given the valued new infusion of performance-based funding investments.

USF continues to work closely with its partners in the Florida Consortium of Metropolitan Research Universities - the University of Central Florida and Florida International University. The group has enjoyed great support from national grant-makers, notably the Helios Foundation. The group's shared best practices have undoubtedly impacted the positive gains USF has made in its student success metrics, and we anticipate only further improvement in the future. In the coming months, the Consortium plans to hire an executive director and team dedicated to exploring ways to share services or ideas for the maximum benefit to students.

Meanwhile, USF continues to build upon its own strong research enterprise, which still boasts the most patents, cumulatively over the last three years, of any state university in Florida by providing the tools and training to innovators on campus so that they are best positioned for success in the marketplace. 


\section{PERFORMANCE FUNDING METRICS}

Reported at the USF System Level 


\section{PREEMINENT RESEARCH UNIVERSITY FUNDING METRICS}

The Board of Governors shall designate each state research university that meets at least 11 of the 12 following academic and research excellence standards as a preeminent state research university. The University of Florida and Florida State University are the only universities required to complete the table below. The Board of Governors will consider the shaded 2015 actual data for approval.

\begin{tabular}{|c|c|c|c|c|c|c|}
\hline & $\begin{array}{l}\text { BENCH- } \\
\text { MARKS }\end{array}$ & $\begin{array}{l}2015 \\
\text { ACTUAL }\end{array}$ & $\begin{array}{l}2016 \\
\text { GOALS }\end{array}$ & $\begin{array}{l}2017 \\
\text { GOALS }\end{array}$ & $\begin{array}{l}2018 \\
\text { GOALS }\end{array}$ & $\begin{array}{c}2019 \\
\text { GOALS }\end{array}$ \\
\hline $\begin{array}{l}\text { Average GPA and SAT Score } \\
\text { for incoming freshman in Fall semester }\end{array}$ & $\begin{array}{l}4.0 \mathrm{GPA} \\
1800 \mathrm{SAT}\end{array}$ & $\begin{array}{c}4.0 \\
1770 \\
\text { Fall } 2014\end{array}$ & $\begin{array}{c}4.05 \\
1800 \\
\text { Fall } 2015\end{array}$ & $\begin{array}{c}4.08 \\
\mathrm{n} / \mathrm{a} \\
\text { Fall } 2016\end{array}$ & $\begin{array}{c}4.1 \\
\mathrm{n} / \mathrm{a} \\
\text { Fall } 2017\end{array}$ & $\begin{array}{c}4.1 \\
\mathrm{n} / \mathrm{a} \\
\text { Fall } 2018\end{array}$ \\
\hline $\begin{array}{l}\text { Public University National Ranking } \\
\text { (in more than one national ranking) }\end{array}$ & Top 50 & $\begin{array}{c}3 \\
2015\end{array}$ & $\begin{array}{c}3 \\
2016\end{array}$ & $\begin{array}{c}3 \\
2017\end{array}$ & $\begin{array}{c}4 \\
2018\end{array}$ & $\begin{array}{c}4 \\
2019\end{array}$ \\
\hline $\begin{array}{l}\text { Freshman Retention Rate } \\
\text { (Full-time, FTIC) }\end{array}$ & $90 \%$ & $\begin{array}{l}89 \% \\
2013-14\end{array}$ & $\begin{array}{l}90 \% \\
2014-15\end{array}$ & $\begin{array}{l}91 \% \\
2015-16\end{array}$ & $\begin{array}{l}91 \% \\
2016-17\end{array}$ & $\begin{array}{l}91 \% \\
2017-18\end{array}$ \\
\hline $\begin{array}{l}\text { 6-year Graduation Rate } \\
\text { (Full-time, FTIC) }\end{array}$ & $70 \%$ & $\begin{array}{l}67 \% \\
2008-14\end{array}$ & $\begin{array}{l}68 \% \\
2009-15\end{array}$ & $\begin{array}{l}63 \% \\
2010-16\end{array}$ & $\begin{array}{l}70 \% \\
2011-17\end{array}$ & $\begin{array}{l}72 \% \\
2012-18\end{array}$ \\
\hline National Academy Memberships & 6 & $\begin{array}{c}3 \\
2012\end{array}$ & $\begin{array}{c}3 \\
2013\end{array}$ & $\begin{array}{c}6 \\
2014\end{array}$ & $\begin{array}{c}7 \\
2015\end{array}$ & $\begin{array}{c}8 \\
2016\end{array}$ \\
\hline $\begin{array}{l}\text { Total Annual Research Expenditures (\$M) } \\
\text { (Science \& Engineering only) }\end{array}$ & $\$ 200 M$ & $\begin{array}{l}\$ 437 \\
2013-14\end{array}$ & $\begin{array}{l}\$ 438 \\
2014-15\end{array}$ & $\begin{array}{l}\$ 439 \\
2015-16\end{array}$ & $\begin{array}{l}\$ 440 \\
2016-17\end{array}$ & $\begin{array}{l}\$ 441 \\
2017-18\end{array}$ \\
\hline $\begin{array}{l}\text { Total Annual Research Expenditures } \\
\text { in Diversified Non-Medical Sciences (\$M) } \\
\text { (Science \& Engineering only) }\end{array}$ & $\$ 150 M$ & $\begin{array}{l}\$ 238 \\
2013-14\end{array}$ & $\begin{array}{l}\$ 239 \\
2014-15\end{array}$ & $\begin{array}{l}\$ 240 \\
2015-16\end{array}$ & $\begin{array}{l}\$ 241 \\
2016-17\end{array}$ & $\begin{array}{l}\$ 242 \\
2017-18\end{array}$ \\
\hline $\begin{array}{l}\text { National Ranking in S.T.E.M. } \\
\text { Research Expenditures } \\
\text { (includes public \& private institutions) }\end{array}$ & $\begin{array}{l}\text { Top } 100 \\
\text { in } 5 \text { of } 8 \\
\text { disciplines }\end{array}$ & $\begin{array}{c}7 \\
2012-13\end{array}$ & $\begin{array}{c}7 \\
2013-14\end{array}$ & $\begin{array}{c}8 \\
2014-15\end{array}$ & $\begin{array}{c}8 \\
2015-16\end{array}$ & $\begin{array}{c}8 \\
2016-17\end{array}$ \\
\hline $\begin{array}{l}\text { Patents Awarded } \\
\text { (over } 3 \text { year period) }\end{array}$ & 100 & $\begin{array}{c}292 \\
2012-14\end{array}$ & $\begin{array}{c}293 \\
2013-15\end{array}$ & $\begin{array}{c}281 \\
2014-16\end{array}$ & $\begin{array}{c}258 \\
2015-17\end{array}$ & $\begin{array}{l}261 \\
2016-18\end{array}$ \\
\hline $\begin{array}{l}\text { Doctoral Degrees Awarded Annually } \\
\text { (excludes Professional degrees) }\end{array}$ & 400 & $\begin{array}{c}330 \\
2013-14\end{array}$ & $\begin{array}{c}315 \\
2014-15\end{array}$ & $\begin{array}{c}320 \\
2015-16\end{array}$ & $\begin{array}{c}325 \\
2016-17\end{array}$ & $\begin{array}{c}330 \\
2017-18\end{array}$ \\
\hline Number of Post-Doctoral Appointees & 200 & $\begin{array}{l}304 \\
\text { Fall } 2011\end{array}$ & $\begin{array}{c}289 \\
\text { Fall } 2012\end{array}$ & $\begin{array}{l}321 \\
\text { Fall } 2013\end{array}$ & $\begin{array}{l}298 \\
\text { Fall } 2014\end{array}$ & $\begin{array}{l}290 \\
\text { Fall } 2015\end{array}$ \\
\hline Endowment Size (\$M) & $\$ 500 M$ & $\begin{array}{l}\$ 417 \\
2013-14\end{array}$ & $\begin{array}{l}\$ 420 \\
2014-15\end{array}$ & $\begin{array}{l}\$ 450 \\
2015-16\end{array}$ & $\begin{array}{l}\$ 485 \\
2016-17\end{array}$ & $\begin{array}{l}\$ 525 \\
2017-18\end{array}$ \\
\hline $\begin{array}{l}\text { NUMBER OF METRICS } \\
\text { ABOVE THE BENCHMARK }\end{array}$ & 11 of 12 & 6 & 8 & 8 & 9 & 10 \\
\hline
\end{tabular}

Note: Due to the various timelines that these metrics represent, the data reported in each column corresponds to the most updated data for the June Board meeting each year. Metrics are defined in appendix. For more information about Preeminent state research universities, see 1001.7065 Florida Statutes. 


\section{KEY PERFORMANCE INDICATORS}

The Board of Governors has selected the following Key Performance Indicators from its 2025 System Strategic Plan and from accountability metrics identified by the Florida Legislature. The Key Performance Indicators emphasize three primary areas of focus: Academic Quality, Operational Efficiency, and Return on Investment. The indicators address common goals across all universities while also providing flexibility to address institution-specific goals from a list of metrics in the 2025 System Strategic Plan.

The Goals Specific to Research Universities apply only to those universities classified by the Carnegie Foundation for the Advancement of Teaching as being a 'Research University ${ }^{\prime}$, which includes Florida A\&M University (by university request), Florida Atlantic University, Florida International University, Florida State University, University of Central Florida, University of Florida, and the University of South Florida.

1 The Carnegie Foundation for the Advancement of Teaching has developed a well-respected system of categorizing postsecondary institutions that includes consideration of each doctorate-granting university's research activities - for more information see link. 


\section{KEY PERFORMANCE INDICATORS}

\section{Metrics Common to All Universities}

\begin{tabular}{|c|c|c|c|c|c|c|}
\hline & $\begin{array}{l}\text { FIVE YEAR } \\
\text { TREND }\end{array}$ & $\begin{array}{c}2015 \\
\text { ACTUAL }\end{array}$ & $\begin{array}{c}2016 \\
\text { GOALS }\end{array}$ & $\begin{array}{c}2017 \\
\text { GOALS }\end{array}$ & $\begin{array}{c}2018 \\
\text { GOALS }\end{array}$ & $\begin{array}{c}2019 \\
\text { GOALS }\end{array}$ \\
\hline \multicolumn{7}{|l|}{ Academic Quality } \\
\hline $\begin{array}{l}\text { National Rankings } \\
\text { for University }\end{array}$ & $\mathrm{n} / \mathrm{a}$ & $\begin{array}{c}3 \\
2015\end{array}$ & $\begin{array}{c}3 \\
2016\end{array}$ & $\begin{array}{c}3 \\
2017\end{array}$ & $\begin{array}{c}4 \\
2018\end{array}$ & $\begin{array}{c}4 \\
2019\end{array}$ \\
\hline $\begin{array}{l}\text { SAT Score* } \\
\text { [for } 3 \text { subtests] }\end{array}$ & $35 \Delta$ & $\begin{array}{l}1770 \\
\text { Fall } 2014\end{array}$ & $\begin{array}{l}1800 \\
\text { Fall } 2015\end{array}$ & $\mathrm{n} / \mathrm{a}$ & $\mathrm{n} / \mathrm{a}$ & $\mathrm{n} / \mathrm{a}$ \\
\hline High School GPA & $0.1 \Delta$ & $\begin{array}{c}4.0 \\
\text { Fall } 2014\end{array}$ & $\begin{array}{c}4.1 \\
\text { Fall } 2015\end{array}$ & $\begin{array}{c}4.1 \\
\text { Fall } 2016\end{array}$ & $\begin{array}{c}4.1 \\
\text { Fall } 2017\end{array}$ & $\begin{array}{c}4.1 \\
\text { Fall } 2018\end{array}$ \\
\hline $\begin{array}{l}\text { Professional/Licensure Exam } \\
\text { First-time Pass Rates }{ }^{1} \\
\text { Exams Above Benchmarks } \\
\text { Exams Below Benchmarks }\end{array}$ & $\begin{array}{l}\mathrm{n} / \mathrm{a} \\
\mathrm{n} / \mathrm{a}\end{array}$ & $\begin{array}{c}3 \\
2 \\
2013-14\end{array}$ & $\begin{array}{c}5 \\
0 \\
2014-15\end{array}$ & $\begin{array}{c}6 \\
0 \\
2015-16\end{array}$ & $\begin{array}{c}6 \\
0 \\
2016-17\end{array}$ & $\begin{array}{c}6 \\
0 \\
2017-18\end{array}$ \\
\hline \multicolumn{7}{|l|}{ Operational Efficiency } \\
\hline Freshman Retention Rate ${ }^{* *} 1$ & $0.5 \mathrm{pts}$ & $\begin{array}{l}89 \% \\
2013-14\end{array}$ & $\begin{array}{l}89 \% \\
2014-15\end{array}$ & $\begin{array}{l}90 \% \\
2015-16\end{array}$ & $\begin{array}{l}91 \% \\
2016-17\end{array}$ & $\begin{array}{l}91 \% \\
2017-18\end{array}$ \\
\hline $\begin{array}{l}\text { FTIC Graduation Rates ** } 2 \\
\text { In } 4 \text { years (or less) }\end{array}$ & $15.5 \mathrm{pts}$ & $\begin{array}{l}44 \% \\
2010-14\end{array}$ & $\begin{array}{l}46 \% \\
2011-15\end{array}$ & $\begin{array}{l}48 \% \\
2012-16\end{array}$ & $\begin{array}{l}50 \% \\
2013-17\end{array}$ & $\begin{array}{l}52 \% \\
2014-18\end{array}$ \\
\hline In 6 years (or less) & $15.9 \mathrm{pts}$ & $\begin{array}{c}67 \% \\
2008-14\end{array}$ & $\begin{array}{l}68 \% \\
2009-15\end{array}$ & $\begin{array}{c}63 \% \\
2010-16\end{array}$ & $\begin{array}{l}70 \% \\
2011-17\end{array}$ & $\begin{array}{l}72 \% \\
2012-18\end{array}$ \\
\hline $\begin{array}{l}\text { AA Transfer Graduation Rates } \\
\text { In } 2 \text { years (or less) }\end{array}$ & $3.8 \mathrm{pts}$ & $\begin{array}{c}31 \% \\
2012-14\end{array}$ & $\begin{array}{l}31 \% \\
2013-15\end{array}$ & $\begin{array}{l}32 \% \\
2014-16\end{array}$ & $\begin{array}{l}33 \% \\
2015-17\end{array}$ & $\begin{array}{c}34 \% \\
2016-18\end{array}$ \\
\hline $\begin{array}{l}\text { FTIC Average Time to Degree } \\
\text { (in years) }\end{array}$ & $-0.5 \Delta$ & $\begin{array}{c}4.1 \\
2013-14\end{array}$ & $\begin{array}{c}4.1 \\
2014-15\end{array}$ & $\begin{array}{c}4.1 \\
2015-16\end{array}$ & $\begin{array}{c}4.1 \\
2016-17\end{array}$ & $\begin{array}{c}4.1 \\
2017-18\end{array}$ \\
\hline \multicolumn{7}{|l|}{ Return on Investment } \\
\hline $\begin{array}{l}\text { Bachelor's Degrees Awarded } \\
\text { First Majors Only }\end{array}$ & $18 \% \Delta$ & $\begin{array}{l}8,079 \\
2013-14\end{array}$ & $\begin{array}{l}8,200 \\
2014-15\end{array}$ & $\begin{array}{l}8,250 \\
2015-16\end{array}$ & $\begin{array}{l}8,400 \\
2016-17\end{array}$ & $\begin{array}{l}8,550 \\
2017-18\end{array}$ \\
\hline $\begin{array}{l}\text { Percent of Bachelor's Degrees } \\
\text { in STEM \& Health }\end{array}$ & 9.4 pts & $\begin{array}{l}37 \% \\
2013-14\end{array}$ & $\begin{array}{c}38 \% \\
2014-15\end{array}$ & $\begin{array}{l}46 \% \\
2015-16\end{array}$ & $\begin{array}{l}49 \% \\
2016-17\end{array}$ & $\begin{array}{l}50 \% \\
2017-18\end{array}$ \\
\hline Graduate Degrees Awarded & $16 \% \Delta$ & $\begin{array}{l}3,181 \\
2013-14\end{array}$ & $\begin{array}{l}3,300 \\
2014-15\end{array}$ & $\begin{array}{l}3,450 \\
2015-16\end{array}$ & $\begin{array}{l}3,600 \\
2016-17\end{array}$ & $\begin{array}{l}3,750 \\
2017-18\end{array}$ \\
\hline $\begin{array}{l}\text { Percent of Graduate Degrees } \\
\text { in STEM \& Health }\end{array}$ & $11.2 \mathrm{pts}$ & $\begin{array}{l}56 \% \\
2013-14\end{array}$ & $\begin{array}{l}57 \% \\
2014-15\end{array}$ & $\begin{array}{l}58 \% \\
2015-16\end{array}$ & $\begin{array}{l}60 \% \\
2016-17\end{array}$ & $\begin{array}{l}61 \% \\
2017-18\end{array}$ \\
\hline $\begin{array}{l}\text { Annual Gifts Received } \\
\text { (\$Millions) }\end{array}$ & $\% \Delta$ & \multicolumn{5}{|c|}{ Reported at the USF System level } \\
\hline $\begin{array}{l}\text { Endowment } \\
\text { (\$Millions) }\end{array}$ & $\% \Delta$ & \multicolumn{5}{|c|}{ Reported at the USF System level } \\
\hline
\end{tabular}

Note*: The College Board is revising the SAT test starting March 2016.

${ }^{* *} \mathrm{FTIC}$ retention and graduation rate percentages represent students starting and ending at USF Tampa.

$189 \%$ of USF Tampa students of the 2013 cohort were retained within the USF System

$267 \%$ of USF Tampa students of the 2008 cohort graduated from the USF System within 6-years 


\section{KEY PERFORMANCE INDICATORS}

Metrics Specific to Research Universities

\begin{tabular}{|c|c|c|c|c|c|c|}
\hline & $\begin{array}{l}\text { FIVE YEAR } \\
\text { TREND }\end{array}$ & $\begin{array}{c}2015 \\
\text { ACTUAL }\end{array}$ & $\begin{array}{c}2016 \\
\text { GOALS }\end{array}$ & $\begin{array}{l}2017 \\
\text { GOALS }\end{array}$ & $\begin{array}{l}2018 \\
\text { GOALS }\end{array}$ & $\begin{array}{c}2019 \\
\text { GOALS }\end{array}$ \\
\hline \multicolumn{7}{|l|}{ Academic Quality } \\
\hline Faculty Awards & $-30 \% \Delta$ & $\begin{array}{c}7 \\
2012\end{array}$ & $\begin{array}{c}8 \\
2013\end{array}$ & $\begin{array}{c}9 \\
2014\end{array}$ & $\begin{array}{r}10 \\
2015\end{array}$ & $\begin{array}{c}11 \\
2016\end{array}$ \\
\hline National Academy Members & $100 \% \Delta$ & $\begin{array}{c}3 \\
2012\end{array}$ & $\begin{array}{c}3 \\
2013\end{array}$ & $\begin{array}{c}6 \\
2014\end{array}$ & $\begin{array}{c}7 \\
2015\end{array}$ & $\begin{array}{c}8 \\
2016\end{array}$ \\
\hline $\begin{array}{l}\text { Number of Post-Doctoral } \\
\text { Appointees }\end{array}$ & $23 \% \Delta$ & $\begin{array}{l}321 \\
\text { Fall } 2013\end{array}$ & $\begin{array}{l}298 \\
\text { Fall } 2014\end{array}$ & $\begin{array}{l}290 \\
\text { Fall } 2015\end{array}$ & $\begin{array}{l}295 \\
\text { Fall } 2016\end{array}$ & $\begin{array}{l}300 \\
\text { Fall } 2017\end{array}$ \\
\hline $\begin{array}{l}\text { Number of Science \& Engineering } \\
\text { Disciplines Nationally Ranked in } \\
\text { Top } 100 \text { for Research } \\
\text { Expenditures }\end{array}$ & $\mathrm{n} / \mathrm{a}$ & $\begin{array}{l}7 \text { of } 8 \\
2012-13\end{array}$ & $\begin{array}{l}7 \text { of } 8 \\
2013-14\end{array}$ & $\begin{array}{l}8 \text { of } 8 \\
2014-15\end{array}$ & $\begin{array}{l}8 \text { of } 8 \\
2015-16\end{array}$ & $\begin{array}{l}8 \text { of } 8 \\
2016-17\end{array}$ \\
\hline \multicolumn{7}{|l|}{ Return on Investment } \\
\hline $\begin{array}{l}\text { Total Research Expenditures (\$M) } \\
\text { [includes non-Science \& Engineering } \\
\text { disciplines] }\end{array}$ & $27 \% \Delta$ & $\begin{array}{l}\$ 489 \\
2013-14\end{array}$ & $\begin{array}{l}\$ 490 \\
2014-15\end{array}$ & $\begin{array}{l}\$ 491 \\
2015-16\end{array}$ & $\begin{array}{l}\$ 492 \\
2016-17\end{array}$ & $\begin{array}{l}\$ 493 \\
2017-18\end{array}$ \\
\hline $\begin{array}{l}\text { Science \& Engineering Research } \\
\text { Expenditures (\$M) }\end{array}$ & $32 \% \Delta$ & $\begin{array}{l}\$ 437 \\
2013-14\end{array}$ & $\begin{array}{l}\$ 438 \\
2014-15\end{array}$ & $\begin{array}{l}\$ 439 \\
2015-16\end{array}$ & $\begin{array}{l}\$ 440 \\
2016-17\end{array}$ & $\begin{array}{l}\$ 441 \\
2017-18\end{array}$ \\
\hline $\begin{array}{l}\text { Science \& Engineering R\&D } \\
\text { Expenditures in Non- } \\
\text { Medical/Health Sciences (\$M) }\end{array}$ & $83 \% \Delta$ & $\begin{array}{l}\$ 238 \\
2013-14\end{array}$ & $\begin{array}{l}\$ 239 \\
2014-15\end{array}$ & $\begin{array}{l}\$ 240 \\
2015-16\end{array}$ & $\begin{array}{l}\$ 241 \\
2016-17\end{array}$ & $\begin{array}{l}\$ 242 \\
2017-18\end{array}$ \\
\hline $\begin{array}{l}\text { Percent of Research Expenditures } \\
\text { funded from External Sources }\end{array}$ & $-10 \mathrm{pts}$ & $\begin{array}{l}60 \% \\
2013-14\end{array}$ & $\begin{array}{l}59 \% \\
2014-15\end{array}$ & $\begin{array}{l}60 \% \\
2015-16\end{array}$ & $\begin{array}{l}61 \% \\
2016-17\end{array}$ & $\begin{array}{l}62 \% \\
2017-18\end{array}$ \\
\hline Patents Issued & $139 \% \Delta$ & $\begin{array}{l}110 \\
2014\end{array}$ & $\begin{array}{l}85 \\
2015\end{array}$ & $\begin{array}{l}86 \\
2016\end{array}$ & $\begin{array}{l}87 \\
2017\end{array}$ & $\begin{array}{l}88 \\
2018\end{array}$ \\
\hline Licenses/Options Executed & $200 \% \Delta$ & $\begin{array}{c}75 \\
2012-13\end{array}$ & $\begin{array}{c}91 \\
2013-14\end{array}$ & $\begin{array}{c}92 \\
2014-15\end{array}$ & $\begin{array}{c}93 \\
2015-16\end{array}$ & $\begin{array}{c}94 \\
2016-17\end{array}$ \\
\hline Licensing Income Received (\$M) & $38 \% \Delta$ & $\begin{array}{l}\$ 1.8 \\
2012-13\end{array}$ & $\begin{array}{l}\$ 1.4 \\
2013-14\end{array}$ & $\begin{array}{l}\$ 1.8 \\
2014-15\end{array}$ & $\begin{array}{l}\$ 1.9 \\
2015-16\end{array}$ & $\begin{array}{l}\$ 2.0 \\
2016-17\end{array}$ \\
\hline Number of Start-up Companies & $200 \% \Delta$ & $\begin{array}{c}9 \\
2012-13\end{array}$ & $\begin{array}{c}11 \\
2013-14\end{array}$ & $\begin{array}{c}9 \\
2014-15\end{array}$ & $\begin{array}{c}9 \\
2015-16\end{array}$ & $\begin{array}{c}10 \\
2016-17\end{array}$ \\
\hline $\begin{array}{l}\text { National Rank is Higher than } \\
\text { Predicted by the Financial } \\
\text { Resources Ranking } \\
\text { [based on U.S. News \& World Report] }\end{array}$ & $\mathrm{n} / \mathrm{a}$ & $\begin{array}{l}\frac{161}{171} \\
2015\end{array}$ & $\begin{array}{l}\frac{158}{171} \\
2016\end{array}$ & $\begin{array}{l}\frac{157}{170} \\
2017\end{array}$ & $\begin{array}{l}\frac{156}{169} \\
2018\end{array}$ & $\begin{array}{l}\frac{155}{168} \\
2019\end{array}$ \\
\hline $\begin{array}{l}\text { Research Doctoral } \\
\text { Degrees Awarded }\end{array}$ & $35 \% \Delta$ & $\begin{array}{c}330 \\
2013-14\end{array}$ & $\begin{array}{c}315 \\
2014-15\end{array}$ & $\begin{array}{c}320 \\
2015-16\end{array}$ & $\begin{array}{c}325 \\
2016-17\end{array}$ & $\begin{array}{c}330 \\
2017-18\end{array}$ \\
\hline $\begin{array}{l}\text { Professional Doctoral } \\
\text { Degrees Awarded }\end{array}$ & $38 \% \Delta$ & $\begin{array}{c}216 \\
2013-14\end{array}$ & $\begin{array}{c}327 \\
2014-15\end{array}$ & $\begin{array}{c}387 \\
2015-16\end{array}$ & $\begin{array}{c}365 \\
2016-17\end{array}$ & $\begin{array}{c}339 \\
2017-18\end{array}$ \\
\hline $\begin{array}{l}\text { TOTAL NUMBER OF } \\
\text { IMPROVING METRICS }\end{array}$ & & & 9 & 13 & 11 & 13 \\
\hline
\end{tabular}




\section{KEY PERFORMANCE INDICATORS}

\section{Institution Specific Goals}

Each university will provide updates for the metric goals reported in last year's Work Plans. The Board of Governors will consider the shaded 2017 goals for approval. University leadership will need to discuss any proposed changes with Board of Governors staff.

\begin{tabular}{|c|c|c|c|c|c|c|}
\hline & $\begin{array}{c}\text { FIVE YEAR } \\
\text { TREND } \\
(09 / 10- \\
13 / 14)\end{array}$ & $\begin{array}{c}2015 \\
\text { ACTUAL }\end{array}$ & $\begin{array}{l}2016 \\
\text { GOALS }\end{array}$ & $\begin{array}{l}2017 \\
\text { GOALS }\end{array}$ & $\begin{array}{c}2018 \\
\text { GOALS }\end{array}$ & $\begin{array}{l}2019 \\
\text { GOALS }\end{array}$ \\
\hline $\begin{array}{l}\text { Graduate Degrees in Areas of } \\
\text { Strategic Emphasis }\end{array}$ & $31.4 \% \Delta$ & $\begin{array}{l}2,275 \\
2013-14\end{array}$ & $\begin{array}{l}2,400 \\
2014-15\end{array}$ & $\begin{array}{l}2,436 \\
2015-16\end{array}$ & $\begin{array}{l}2,550 \\
2016-17\end{array}$ & $\begin{array}{l}2,600 \\
2017-18\end{array}$ \\
\hline $\begin{array}{l}\text { Freshman in Top } 10 \% \text { of } \\
\text { Graduating High School Class }\end{array}$ & $0.2 \mathrm{pts} \Delta$ & $\begin{array}{l}36 \% \\
2013-14\end{array}$ & $\begin{array}{l}30 \% \\
2014-15\end{array}$ & $\begin{array}{l}36 \% \\
2015-16\end{array}$ & $\begin{array}{l}37 \% \\
2016-17\end{array}$ & $\begin{array}{l}38 \% \\
2017-18\end{array}$ \\
\hline $\begin{array}{l}\text { Percent of Course Sections } \\
\text { Offered via Distance and Blended } \\
\text { Learning }\end{array}$ & $2.3 \mathrm{pts} \Delta$ & $\begin{array}{c}11 \% \\
2013-14\end{array}$ & $\begin{array}{c}12 \% \\
2014-15\end{array}$ & $\begin{array}{c}12 \% \\
2015-16\end{array}$ & $\begin{array}{l}12 \% \\
2016-17\end{array}$ & $\begin{array}{l}13 \% \\
2017-18\end{array}$ \\
\hline
\end{tabular}

To further distinguish the university's distinctive mission, the university may choose to provide two additional narrative and metric goals that are based on the university's own strategic plan.

Goal 1. As an RU/VH institution, with a strategic goal of engaging in high impact research, USF will continue to build a sustainable research infrastructure, including total research expenditures as defined by the National Science Foundation.

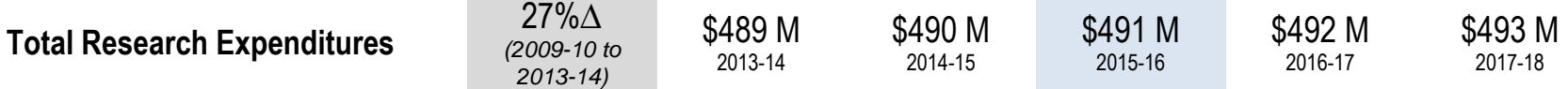

Goal 2. As an RU/VH institution, with a strategic goal of engaging in high impact research, USF will continue to build a sustainable research infrastructure, including federal research expenditures as defined by the National Science Foundation

Federal Research Expenditures 


\section{FISCAL INFORMATION}

University Revenues (in Millions of Dollars)

\begin{tabular}{lcc} 
& $\begin{array}{c}\mathbf{2 0 1 4 - 1 5} \\
\text { Actual }\end{array}$ & $\begin{array}{c}\text { 2015-16 } \\
\text { Appropriations }\end{array}$ \\
\hline Education \& General - Main Operations & & \\
\hline State Funds & $\$ 240.0$ & Pending \\
\hline Tuition & $\$ 163.7$ & Pending \\
\hline TOTAL MAIN OPERATIONS & $\$ 403.7$ & Pending \\
\hline Education \& General - Health-Science Center / Medical Schools & & \\
\hline State Funds & $\$ 74.6$ & Pending \\
\hline Tuition & $\$ 56.0$ & Pending \\
\hline TOTAL HSC & $\$ 130.6$ & Pending \\
\hline$\quad$ EDUCATION \& GENERAL TOTAL REVENUES & $\$ 534.30$ & Pending \\
\hline
\end{tabular}

Note: State funds include General Revenue funds, Lottery funds, Federal Stimulus funds, and Phosphate Research funds (for Polytechnic) appropriated by the Florida Legislature (as reported in the Annual Accountability Report). Actual tuition includes base tuition and tuition differential fee revenues for resident and non-resident undergraduate and graduate students net of waivers (as reported in the Annual Accountability Report). Actual tuition revenues are not yet available for the 2013-14 year.

\section{OTHER BUDGET ENTITIES}

\section{Auxiliary Enterprises}

Resources associated with auxiliary units that are self supporting through fees, payments and charges. Examples include housing, food services, bookstores, parking services, health centers.
Revenues
$\$ 193.38$
Pending

\section{Contracts \& Grants}

Resources received from federal, state or private sources for the purposes of conducting research and public service activities.

\begin{tabular}{lcc}
\hline \multicolumn{1}{c}{ Revenues } & $\$ 282.61$ & Pending \\
\hline $\begin{array}{l}\text { Local Funds } \\
\text { Resources associated with student activity (supported by the student activity fee), student financial aid, concessions, intercollegiate } \\
\text { athletics, technology fee, green fee, and student life \& services fee. }\end{array}$ & $\$ 438.82$ & Pending \\
\hline \multicolumn{1}{l}{ Revenues } \\
$\begin{array}{l}\text { Faculty Practice Plans } \\
\text { Revenues/receipts are funds generated from faculty practice plan activities. }\end{array}$
\end{tabular}




\section{FISCAL INFORMATION (continued)}

\section{Undergraduate Resident Tuition Summary (for 30 credithours)}

\begin{tabular}{|c|c|c|c|c|c|}
\hline & $\begin{array}{c}\text { FY 2012-13 } \\
\text { ACTUAL }\end{array}$ & $\begin{array}{c}\text { FY 2013-14 } \\
\text { ACTUAL }\end{array}$ & $\begin{array}{c}\text { FY 2014-15 } \\
\text { ACTUAL }\end{array}$ & $\begin{array}{c}\text { FY 2015-16 } \\
\text { REQUEST }\end{array}$ & $\begin{array}{l}\text { FY 2016-17 } \\
\text { PLANNED } \\
\end{array}$ \\
\hline Base Tuition & $\$ 3,100$ & $\$ 3,152$ & $\$ 3,152$ & $\$ 3,152$ & $\$ 3,152$ \\
\hline Tuition Differential Fee & $\$ 1,406$ & $\$ 1,406$ & $\$ 1,406$ & $\$ 1,406$ & $\$ 1,406$ \\
\hline Percent Increase & $11 \%$ & $1.70 \%$ & $0.00 \%$ & $0.00 \%$ & $0.00 \%$ \\
\hline Required Fees ${ }^{1}$ & $\$ 1,828$ & $\$ 1,851$ & $\$ 1,851$ & $\$ 1,851$ & $\$ 1,851$ \\
\hline TOTAL TUITION AND FEES & $\$ 6,334$ & $\$ 6,410$ & $\$ 6,410$ & $\$ 6,410$ & $\$ 6,410$ \\
\hline
\end{tabular}

Note': For more information regarding required fees see list of per credit hour fees and block fees on next page.

\section{Student Debt Summary}

\begin{tabular}{lccccc} 
& $\begin{array}{c}2010-11 \\
\text { ACTUAL }\end{array}$ & $\begin{array}{c}2011-12 \\
\text { ACTUAL }\end{array}$ & $\begin{array}{c}2012-13 \\
\text { ACTUAL }\end{array}$ & $\begin{array}{c}2013-14 \\
\text { ACTUAL }\end{array}$ & $\begin{array}{c}2014-15 \\
\text { GOAL }\end{array}$ \\
\hline Percent of Bachelor's Recipients with Debt & $53 \%$ & $57 \%$ & $59 \%$ & $60 \%$ & $59 \%$ \\
$\begin{array}{l}\text { Average Amount of Debt } \\
\text { for Bachelor's who have graduated with debt }\end{array}$ & $\$ 21,784$ & $\$ 22,623$ & $\$ 22,719$ & $\$ 22,719$ & $\$ 22,000$ \\
& 2009 & 2010 & 2011 & 2012 & 2013 \\
NSLDS Cohort Year & $10.1 \%$ & $9.0 \%$ & $7.5 \%$ & $5.4 \%$ est & $5.3 \%$
\end{tabular}

Cost of Attendance (for Full-Time Undergraduate Florida Residents in the Fall and Spring of 2014-15)

\begin{tabular}{ccccccc} 
& $\begin{array}{c}\text { TUITION } \\
\& \text { FEES }\end{array}$ & $\begin{array}{c}\text { BOOKS } \& \\
\text { SUPPLIES }\end{array}$ & $\begin{array}{c}\text { ROOM } \\
\& \text { BOARD }\end{array}$ & TRANSPORTATION & $\begin{array}{c}\text { OTHER } \\
\text { EXPENSES }\end{array}$ & TOTAL \\
\hline ON-CAMPUS & $\$ 6,410$ & $\$ 1,000$ & $\$ 9,400$ & $\$ 1,500$ & $\$ 2,600$ & $\$ 20,910$ \\
\hline AT HOME & $\$ 6,410$ & $\$ 1,000$ & $\$ 4,700$ & $\$ 1,500$ & $\$ 2,600$ & $\$ 16,210$ \\
\hline
\end{tabular}

Estimated Net Cost by Family Income (for Full-Time Undergraduate Florida Residents in the Fall and Spring of 2014-15)

\begin{tabular}{|c|c|c|c|c|c|c|}
\hline \multirow{2}{*}{$\begin{array}{l}\text { FAMILY } \\
\text { INCOME } \\
\text { GROUPS }\end{array}$} & \multicolumn{2}{|c|}{$\begin{array}{l}\text { FULL-TIME RESIDENT } \\
\text { UNDERGRADUATES }\end{array}$} & \multirow{2}{*}{$\begin{array}{c}\text { AVG. NET } \\
\text { COST OF } \\
\text { ATTENDANCE }\end{array}$} & \multirow{2}{*}{$\begin{array}{c}\text { AVG. NET } \\
\text { TUITION } \\
\text { \& FEES }\end{array}$} & \multirow{2}{*}{$\begin{array}{c}\text { AVG. } \\
\text { GIFT AID } \\
\text { AMOUNT }\end{array}$} & \multirow{2}{*}{$\begin{array}{r}\text { AVG. } \\
\text { LOAN } \\
\text { AMOUNT }\end{array}$} \\
\hline & HEADCOUNT & PERCENT & & & & \\
\hline Below $\$ 40,000$ & 5,883 & $34 \%$ & $\$ 11,268$ & $-\$ 2,913$ & $\$ 8,839$ & $\$ 3,978$ \\
\hline$\$ 40,000-\$ 59,999$ & 2,117 & $12 \%$ & $\$ 13,126$ & $-\$ 899$ & $\$ 6,902$ & $\$ 3,661$ \\
\hline$\$ 60,000-\$ 79,999$ & 1,599 & $9 \%$ & $\$ 15,555$ & $\$ 1,790$ & $\$ 4,241$ & $\$ 4,147$ \\
\hline$\$ 80,000-\$ 99,999$ & 1,303 & $8 \%$ & $\$ 16,586$ & $\$ 2,770$ & $\$ 3,284$ & $\$ 4,719$ \\
\hline$\$ 100,000$ Above & 4,085 & $24 \%$ & $\$ 16,786$ & $\$ 2,922$ & $\$ 3,158$ & $\$ 3,447$ \\
\hline Missing* & 2,146 & $13 \%$ & $\mathrm{n} / \mathrm{a}$ & $\$ 4,067$ & $\$ 1,974$ & $\$ 155$ \\
\hline TOTAL & 17,133 & $100 \%$ & $\$ 13,954^{*}$ & $\$ 472$ & $\$ 5,534$ & $\$ 3,405$ \\
\hline
\end{tabular}

Notes: This data only represents Fall and Spring financial aid data and is accurate as of March 31, 2015. Please note that small changes to Spring 2014 awards are possible before the data is finalized. Family Income Groups are based on the Total Family Income (including untaxed income) as reported on student FAFSA records. Full-time Students is a headcount based on at least 24 credit hours during Fall and Spring terms. Average Gift Aid includes all grants and scholarships from Federal, State, University and other private sources administered by the Financial Aid Office. Student waivers are also included in the Gift Aid amount. Gift Aid does not include the parental contribution towards EFC. Net Cost of Attendance is the actual average of the total Costs of Attendance (which will vary by income group due to the diversity of students living on- \& off- campus) minus the average Gift Aid amount. Net Tuition \& Fees is the actual average of the total costs of tuition and fees (which will vary by income group due to the amount of credit hours students are enrolled) minus the average Gift Aid amount (see page 16 for list of fees that are included). Average Loan Amount includes Federal (Perkins, Stafford, Ford Direct, and PLUS loans) and all private loans. The bottom-line Average represents the average of all full-time undergraduate Florida residents (note*: the total Net Cost of Attendance does not include students with missing family income data). 'Missing' includes students who did not file a FAFSA. 
 \\ FISCAL INFORMATION (continued) TUITION DIFFERENTIAL FEE INCREASE REQUEST FOR FALL 2015}

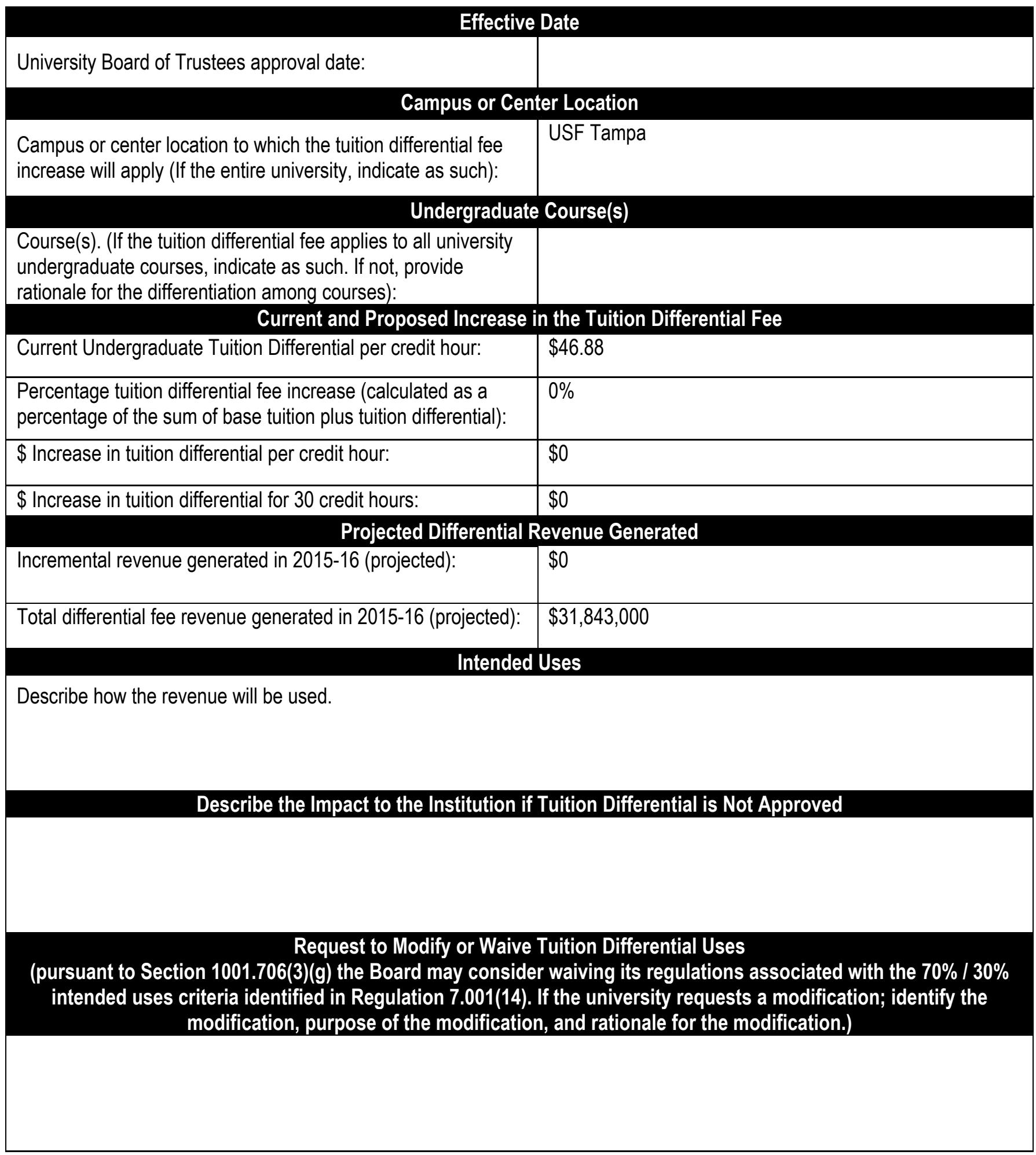




\title{
FISCAL INFORMATION (continued) \\ TUITION DIFFERENTIAL SUPPLEMENTAL INFORMATION
}

Provide the following information for the 2014-15 academic year.

2014-2015 - 60\% Initiatives (list the initiatives provided in

the 2013-14 tuition differential request)

Expanding the high-skilled workforce and assuring job placement, especially in STEM.

Academic Advising and veteran's support.

\author{
University Update on Each Initiative
}

Reorganized and strengthened the Office of Career Services under an Assistant Vice President for Career Services to promote more career training services and internship opportunities for students.

Enhanced academic advising through the development of career ladder for advisors; provided greater professional development opportunities for advisers; implemented a tracking system to support at-risk students and promote first year retention efforts. Contracted with external party to implement predictive analytics platform and deploy sophisticated academic advising tool. Strengthened USF's Veterans' Success program.

Financial counseling and debt reduction

Consolidated Bull2Bull financial education program, using peer educators to promote financial literacy and provide students with personal financial management skills through group and one-onone financial education sessions.

\section{Additional Detail, where applicable}

Total Number of Faculty Hired or Retained (funded by tuition 228 differential):

Total Number of Advisors Hired or Retained (funded by tuition differential):

Total Number of Course Sections Added or Saved (funded by tuition differential):

\section{4-2015 - 40\% Initiatives (list the initiatives provided in} the 2014-15 tuition differential request)

Maintain our need based grant funding commitment to make USF affordable and provide access to low income students.

Provide financial support for talented students with limited income so as to keep average student loan debt flat or with nominal increases.

\section{8}

2474

\section{Additional Information (estimates as of March 19, 2015):}

Unduplicated Count of Students Receiving at least one

Tuition Differential-Funded Award:

\$ Mean (per student receiving an award) of Tuition

Differential-Funded Awards:

\$ Minimum (per student receiving an award) of Tuition Differential-Funded Awards:

\$ Maximum (per student receiving an award) of Tuition

Differential-Funded Awards:

Due to the fact that there were no tuition increases in FY15, we had no additional resources to award. In fact, our available funding for the campus decreased by 1 percent. However, we were able to maintain our awarding strategy to support recruitment and retention activities.

Average debt for the campus is flat.
8759

$\$ 1,214$

$\$ 23$

$\$ 2,500$ 
 \\ FISCAL INFORMATION (continued) \\ TUITION DIFFERENTIAL COLLECTIONS, EXPENDITURES, \& AVAILABLE BALANCES - FISCAL YEAR 2013-14 AND 2014-15}

\section{University Tuition Differential \\ Budget Entity: 48900100 (Educational \& General) \\ SF/Fund: 2 164xxx (Student and Other Fees Trust Fund)}

\section{Estimated Actual}

2014-15

\section{Estimated}

2015-16

\section{FTE Positions:}

Faculty

Advisors

Staff

Total FTE Positions:

\section{Balance Forward from Prior Periods}

Balance Forward

Less: Prior-Year Encumbrances

Beginning Balance Available:

\section{Receipts / Revenues}

Tuition Differential Collections

Interest Revenue - Current Year

Interest Revenue - From Carryforward Balance

Total Receipts / Revenues:

\section{Expenditures}

Salaries \& Benefits

Other Personal Services

Expenses

Operating Capital Outlay

Student Financial Assistance

Expended From Carryforward Balance

${ }^{* *}$ Other Category Expenditures

Total Expenditures:

\section{Ending Balance Available:}
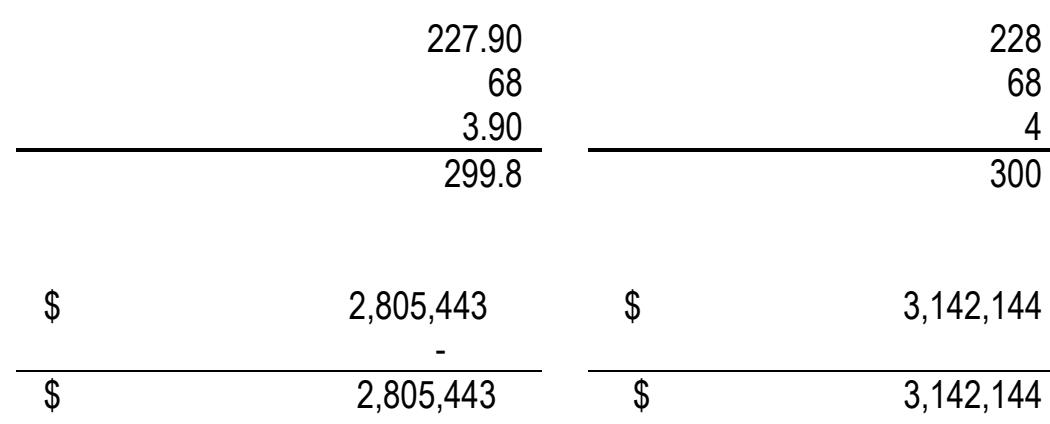

$31,745,663$

97,337

\$ $34,897,261$

$34,985,144$

$\$ 34,985,144$

\begin{tabular}{|c|c|c|c|}
\hline & $\begin{array}{r}20,494,591 \\
640,627 \\
9,892\end{array}$ & & $\begin{array}{r}20,370,000 \\
645,000 \\
10,000\end{array}$ \\
\hline & $10,610,006$ & & $10,556,000$ \\
\hline$\$$ & $31,755,117$ & $\$$ & $34,985,144$ \\
\hline$\$$ & $3,142,144$ & $\$$ & 0 \\
\hline
\end{tabular}

*Since the 2014-15 year has not been completed, provide an estimated actual.

**Provide details for "Other Categories" used. 


\section{FISCAL INFORMATION (continued) \\ UNIVERSITY TUITION, FEES AND HOUSING PROJ ECTIONS}

\section{University of South Florida - Main Campus}

\begin{tabular}{|c|c|c|c|c|c|c|c|}
\hline \multirow[t]{2}{*}{ Undergraduate Students } & \multicolumn{3}{|c|}{ 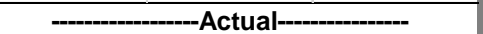 } & \multicolumn{4}{|c|}{ 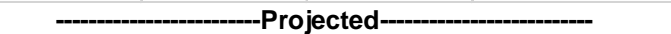 } \\
\hline & 2012-13 & 2013-14 & 2014-15 & 2015-16 & 2016-17 & 2017-18 & 2018-19 \\
\hline \multicolumn{8}{|l|}{ Tuition: } \\
\hline Base Tuition - (projected legislative increase) & $\$ 103.32$ & $\$ 105.07$ & $\$ 105.07$ & $\$ 105.07$ & $\$ 105.07$ & $\$ 105.07$ & $\$ 105.07$ \\
\hline Tuition Differential (no more than 15\%) & $\$ 46.88$ & $\$ 46.88$ & $\$ 46.88$ & $\$ 46.88$ & $\$ 46.88$ & $\$ 46.88$ & $\$ 46.88$ \\
\hline Total Base Tuition and Differential & $\$ 150.20$ & $\$ 151.95$ & $\$ 151.95$ & $\$ 151.95$ & $\$ 151.95$ & $\$ 151.95$ & $\$ 151.95$ \\
\hline$\%$ Change & $11.0 \%$ & $1.2 \%$ & $0.0 \%$ & $0.0 \%$ & $0.0 \%$ & $0.0 \%$ & $0.0 \%$ \\
\hline \multicolumn{8}{|l|}{ Fees (per credit hour): } \\
\hline Student Financial Aid & $\$ 5.16$ & $\$ 5.25$ & $\$ 5.25$ & $\$ 5.25$ & $\$ 5.25$ & $\$ 5.25$ & $\$ 5.25$ \\
\hline Capital Improvement & $\$ 6.76$ & $\$ 6.76$ & $\$ 6.76$ & $\$ 6.76$ & $\$ 6.76$ & $\$ 6.76$ & $\$ 6.76$ \\
\hline Activity \& Service & $\$ 11.71$ & $\$ 12.08$ & $\$ 12.08$ & $\$ 12.08$ & $\$ 12.08$ & $\$ 12.08$ & $\$ 12.08$ \\
\hline Health & $\$ 9.73$ & $\$ 9.94$ & $\$ 10.15$ & $\$ 10.15$ & $\$ 10.15$ & $\$ 10.15$ & $\$ 10.15$ \\
\hline Athletic & $\$ 14.46$ & $\$ 14.46$ & $\$ 14.46$ & $\$ 14.46$ & $\$ 14.46$ & $\$ 14.46$ & $\$ 14.46$ \\
\hline Transportation Access & $\$ 3.00$ & $\$ 3.00$ & $\$ 3.00$ & $\$ 3.00$ & $\$ 3.00$ & $\$ 3.00$ & $\$ 3.00$ \\
\hline Technology $^{1}$ & $\$ 5.16$ & $\$ 5.25$ & $\$ 5.25$ & $\$ 5.25$ & $\$ 5.25$ & $\$ 5.25$ & $\$ 5.25$ \\
\hline Other (list:): Marshall Center & $\$ 1.50$ & $\$ 1.50$ & $\$ 1.50$ & $\$ 1.50$ & $\$ 1.50$ & $\$ 1.50$ & $\$ 1.50$ \\
\hline Student Green Energy & $\$ 1.00$ & $\$ 1.00$ & $\$ 1.00$ & $\$ 1.00$ & $\$ 1.00$ & $\$ 1.00$ & $\$ 1.00$ \\
\hline Sub total (credit hour fees) & $\$ 58.48$ & $\$ 59.24$ & $\$ 59.45$ & $\$ 59.45$ & $\$ 59.45$ & $\$ 59.45$ & $\$ 59.45$ \\
\hline Total Tuition and Fees per credit hour & $\$ 208.68$ & $\$ 211.19$ & $\$ 211.40$ & $\$ 211.40$ & $\$ 211.40$ & $\$ 211.40$ & $\$ 211.40$ \\
\hline$\%$ Change & $9.2 \%$ & $1.2 \%$ & $0.1 \%$ & $0.0 \%$ & $0.0 \%$ & $0.0 \%$ & $0.0 \%$ \\
\hline \multicolumn{8}{|l|}{ Fees (block per term): } \\
\hline Activity \& Service & $\$ 7.00$ & $\$ 7.00$ & $\$ 7.00$ & $\$ 7.00$ & $\$ 7.00$ & $\$ 7.00$ & $\$ 7.00$ \\
\hline \multicolumn{8}{|l|}{ Health } \\
\hline Athletic & $\$ 10.00$ & $\$ 10.00$ & $\$ 10.00$ & $\$ 10.00$ & $\$ 10.00$ & $\$ 10.00$ & $\$ 10.00$ \\
\hline \multicolumn{8}{|l|}{ Transportation Access } \\
\hline Other (list): Marshall Center & $\$ 20.00$ & $\$ 20.00$ & $\$ 20.00$ & $\$ 20.00$ & $\$ 20.00$ & $\$ 20.00$ & $\$ 20.00$ \\
\hline Total Block Fees per term & $\$ 37.00$ & $\$ 37.00$ & $\$ 37.00$ & $\$ 37.00$ & $\$ 37.00$ & $\$ 37.00$ & $\$ 37.00$ \\
\hline$\%$ Change & $0.0 \%$ & $0.0 \%$ & $0.0 \%$ & $0.0 \%$ & $0.0 \%$ & $0.0 \%$ & $0.0 \%$ \\
\hline Total Tuition for 30 Credit Hours & $\$ 4,506.00$ & $\$ 4,558.50$ & $\$ 4,558.50$ & $\$ 4,558.50$ & $\$ 4,558.50$ & $\$ 4,558.50$ & $\$ 4,558.50$ \\
\hline Total Fees for $\mathbf{3 0}$ Credit Hours & $\$ 1,828.40$ & $\$ 1,851.20$ & $\$ 1,857.50$ & $\$ 1,857.50$ & $\$ 1,857.50$ & $\$ 1,857.50$ & $\$ 1,857.50$ \\
\hline Total Tuition and Fees for 30 Credit Hours & $\$ 6,334.40$ & $\$ 6,409.70$ & $\$ 6,416.00$ & $\$ 6,416.00$ & $\$ 6,416.00$ & $\$ 6,416.00$ & $\$ 6,416.00$ \\
\hline \$Change & $\$ 528.60$ & $\$ 75.30$ & $\$ 6.30$ & $\$ 0.00$ & $\$ 0.00$ & $\$ 0.00$ & $\$ 0.00$ \\
\hline$\%$ Change & $9.1 \%$ & $1.2 \%$ & $0.1 \%$ & $0.0 \%$ & $0.0 \%$ & $0.0 \%$ & $0.0 \%$ \\
\hline \multicolumn{8}{|l|}{ Out-of-State Fees } \\
\hline$\overline{\text { Out-of-State Undergraduate Fee }}$ & $\$ 315.00$ & $\$ 346.50$ & $\$ 346.50$ & $\$ 346.50$ & $\$ 346.50$ & $\$ 346.50$ & $\$ 346.50$ \\
\hline Percentage change & $8 \%$ & $10 \%$ & $0 \%$ & $0 \%$ & $0 \%$ & $0 \%$ & $0 \%$ \\
\hline Out-of-State Undergraduate Student Financial Aid ${ }^{3}$ & $\$ 15.75$ & $\$ 17.32$ & $\$ 17.32$ & $\$ 17.32$ & $\$ 17.32$ & $\$ 17.32$ & $\$ 17.32$ \\
\hline Total per credit hour & $\$ 330.75$ & $\$ 363.82$ & $\$ 363.82$ & $\$ 363.82$ & $\$ 363.82$ & $\$ 363.82$ & $\$ 363.82$ \\
\hline \% Change & $8 \%$ & $10 \%$ & $0 \%$ & $0 \%$ & $0 \%$ & $0 \%$ & $0 \%$ \\
\hline Total Tuition for 30 Credit Hours & $\$ 4,506.00$ & $\$ 4,558.50$ & $\$ 4,558.50$ & $\$ 4,558.50$ & $\$ 4,558.50$ & $\$ 4,558.50$ & $\$ 4,558.50$ \\
\hline Total Fees for 30 Credit Hours & $\$ 11,750.90$ & $\$ 12,765.80$ & $\$ 12,765.80$ & $\$ 12,765.80$ & $\$ 12,765.80$ & $\$ 12,765.80$ & $\$ 12,765.80$ \\
\hline Total Tuition and Fees for 30 Credit Hours & $\$ 16,256.90$ & $\$ 17,324.30$ & $\$ 17,324.30$ & $\$ 17,324.30$ & $\$ 17,324.30$ & $\$ 17,324.30$ & $\$ 17,324.30$ \\
\hline \$Change & $\$ 1,263.30$ & $\$ 1,067.40$ & $\$ 0.00$ & $\$ 0.00$ & $\$ 0.00$ & $\$ 0.00$ & $\$ 0.00$ \\
\hline$\%$ Change & $8.4 \%$ & $6.6 \%$ & $0.0 \%$ & $0.0 \%$ & $0.0 \%$ & $0.0 \%$ & $0.0 \%$ \\
\hline Housing/Dining & $\$ 8,960.00$ & $\$ 9,250.00$ & $\$ 9,403.00$ & $\$ 9,403.00$ & $\$ 9,403.00$ & $\$ 9,403.00$ & $\$ 9,403.00$ \\
\hline$\%$ Change & $-4.3 \%$ & $3.2 \%$ & $1.7 \%$ & $0.0 \%$ & $0.0 \%$ & $0.0 \%$ & $0.0 \%$ \\
\hline
\end{tabular}




\section{ENROLLMENT PLANNING}

Planned Enrollment Growth by Student Type (for all E\&G students at all campuses)

\begin{tabular}{|c|c|c|c|c|c|c|c|c|c|}
\hline \multirow{2}{*}{ UNDERGRADUATE } & \multirow[t]{2}{*}{$\begin{array}{l}5 \text { YEAR } \\
\text { TREND } \\
\text { (2009-14) }\end{array}$} & \multicolumn{2}{|c|}{$\begin{array}{c}\text { Fall } 2014 \\
\text { ACTUAL } \\
\text { HEADCOUNT }\end{array}$} & \multicolumn{2}{|c|}{$\begin{array}{c}\text { Fall } 2015 \\
\text { PLANNED } \\
\text { HEADCOUNT }\end{array}$} & \multicolumn{2}{|c|}{$\begin{array}{c}\text { Fall } 2016 \\
\text { PLANNED } \\
\text { HEADCOUNT }\end{array}$} & \multicolumn{2}{|c|}{$\begin{array}{c}\text { Fall } 2017 \\
\text { PLANNED } \\
\text { HEADCOUNT }\end{array}$} \\
\hline & & & & & & & & & \\
\hline FTIC (Regular Admit) & $-1 \% \Delta$ & 15,482 & $50 \%$ & 15,450 & $52 \%$ & 15,500 & $53 \%$ & 15,550 & $53 \%$ \\
\hline FTIC (Profile Admit) & $-33 \% \Delta$ & 183 & $1 \%$ & 120 & $1 \%$ & 100 & $0 \%$ & 100 & $0 \%$ \\
\hline AA Transfers from FCS & $16 \% \Delta$ & 7,195 & $24 \%$ & 9,525 & $32 \%$ & 9,200 & $32 \%$ & 9,300 & $32 \%$ \\
\hline Other Transfers & $-17 \% \Delta$ & 7,458 & $25 \%$ & 4,419 & $15 \%$ & 4,225 & $15 \%$ & 4,300 & $15 \%$ \\
\hline Subtotal & $-2 \% \Delta$ & 30,318 & $100 \%$ & 29,514 & $100 \%$ & 29,025 & $100 \%$ & 29,250 & $100 \%$ \\
\hline \multicolumn{10}{|l|}{ GRADUATE* } \\
\hline Master's & $6 \% \Delta$ & 6,300 & $66 \%$ & 6,350 & $65 \%$ & 6,400 & $65 \%$ & 6,450 & $65 \%$ \\
\hline Research Doctoral & $2 \% \Delta$ & 2,226 & $23 \%$ & 2,375 & $24 \%$ & 2,400 & $24 \%$ & 2,400 & $24 \%$ \\
\hline Professional Doctoral & $74 \% \Delta$ & 1,027 & $11 \%$ & 1,078 & $11 \%$ & 1,106 & $11 \%$ & 1,114 & $11 \%$ \\
\hline Subtotal & $10 \% \Delta$ & 9,553 & $100 \%$ & 9,803 & $100 \%$ & 9,906 & $100 \%$ & 9,964 & $100 \%$ \\
\hline \multicolumn{10}{|l|}{ UNCLASSIFIED } \\
\hline H.S. Dual Enrolled & $-39 \% \Delta$ & 14 & $1 \%$ & 14 & $1 \%$ & 14 & $1 \%$ & 14 & $1 \%$ \\
\hline Other & $3 \% \Delta$ & 1,829 & $99 \%$ & 1,844 & $99 \%$ & 1,858 & $99 \%$ & 1,873 & $99 \%$ \\
\hline Subtotal & $4 \% \Delta$ & 1,843 & $100 \%$ & 1,858 & $100 \%$ & 1,872 & $100 \%$ & 1,887 & $100 \%$ \\
\hline TOTAL & $1 \% \Delta$ & 41,714 & & 41,175 & & 40,803 & & 41,101 & \\
\hline
\end{tabular}

Note*: Includes Medical students.

Planned Enrollment Growth by Method of Instruction (for all E\&G students at all campuses)

\begin{tabular}{|c|c|c|c|c|c|c|c|c|c|}
\hline & \multirow{2}{*}{$\begin{array}{c}3 \text { YEAR } \\
\text { TREND } \\
\text { (2010-11 to } \\
2013-14)\end{array}$} & \multicolumn{2}{|c|}{ 2013-14 } & \multicolumn{2}{|c|}{ 2014-15 } & \multicolumn{2}{|c|}{ 2015-16 } & \multicolumn{2}{|c|}{ 2016-17 } \\
\hline & & $\begin{array}{c}\text { ACTUAL } \\
\text { FTE }\end{array}$ & $\begin{array}{c}\% \text { of } \\
\text { TOTAL }\end{array}$ & $\begin{array}{c}\text { PLANNED } \\
\text { FTE }\end{array}$ & $\begin{array}{c}\% \text { of } \\
\text { TOTAL }\end{array}$ & $\begin{array}{c}\text { PLANNED } \\
\text { FTE }\end{array}$ & $\begin{array}{c}\% \text { of } \\
\text { TOTAL }\end{array}$ & $\begin{array}{c}\text { PLANNED } \\
\text { FTE }\end{array}$ & $\begin{array}{c}\% \text { of } \\
\text { TOTAL }\end{array}$ \\
\hline \multicolumn{10}{|l|}{ UNDERGRADUATE } \\
\hline DISTANCE (>80\%) & $21 \% \Delta$ & 4,072 & $20 \%$ & 4,829 & $24 \%$ & 4,716 & $23 \%$ & 4,726 & $23 \%$ \\
\hline HYBRID (50\%-79\%) & $2 \% \Delta$ & 316 & $2 \%$ & 294 & $1 \%$ & 410 & $2 \%$ & 411 & $2 \%$ \\
\hline TRADITIONAL (<50\%) & $-4 \% \Delta$ & 15,976 & $78 \%$ & 15,338 & $75 \%$ & 15,378 & $75 \%$ & 15,411 & $75 \%$ \\
\hline TOTAL & $0 \% \Delta$ & 20,365 & $100 \%$ & 20,461 & $100 \%$ & 20,504 & $100 \%$ & 20,548 & $100 \%$ \\
\hline \multicolumn{10}{|l|}{ GRADUATE } \\
\hline DISTANCE (80\%) & $-2 \% \Delta$ & 1,041 & $19 \%$ & 1,100 & $20 \%$ & 1,151 & $21 \%$ & 1,171 & $21 \%$ \\
\hline HYBRID (50\%-79\%) & $35 \% \Delta$ & 143 & $3 \%$ & 119 & $2 \%$ & 110 & $2 \%$ & 112 & $2 \%$ \\
\hline TRADITIONAL $(<50 \%)$ & $4 \% \Delta$ & 4,163 & $78 \%$ & 4,205 & $78 \%$ & 4,220 & $77 \%$ & 4,295 & $77 \%$ \\
\hline TOTAL & $4 \% \Delta$ & 5,346 & $100 \%$ & 5,423 & $100 \%$ & 5,480 & $100 \%$ & 5,578 & $100 \%$ \\
\hline
\end{tabular}

Note: Full-time Equivalent (FTE) student is a measure of instructional effort (and student activity) that is based on the number of credit hours that students enroll. FTE is based on the Florida definition, which divides undergraduate credit hours by 40 and graduate credit hours by 32 . Distance Learning is a course in which at least 80 percent of the direct instruction of the course is delivered using some form of technology when the student and instructor are separated by time or space, or both (per 1009.24(17), F.S.). Hybrid is a course where $50 \%$ to $79 \%$ of the instruction is delivered using some form of technology, when the student and instructor are separated by time or space, or both (per SUDS data element 2052). Traditional (and Technology Enhanced) refers to primarily face to face instruction utilizing some form of technology for delivery of supplemental course materials for no more than $49 \%$ of instruction (per SUDS data element 2052).

*Only USF Tampa sections are included in this calculation 


\section{ENROLLMENT PLANNING (continued)}

Planned Enrollment Plan by Residency and Student Level (Florida FTE)

\begin{tabular}{|c|c|c|c|c|c|c|c|c|c|}
\hline & $\begin{array}{c}\text { Estimated } \\
\text { Actual } \\
2014-15 \\
\end{array}$ & $\begin{array}{l}\text { Funded } \\
2015-16 \\
\end{array}$ & $\begin{array}{l}\text { Planned } \\
2015-16\end{array}$ & $\begin{array}{l}\text { Planned } \\
2016-17\end{array}$ & $\begin{array}{l}\text { Planned } \\
2017-18\end{array}$ & $\begin{array}{l}\text { Planned } \\
2018-19 \\
\end{array}$ & $\begin{array}{c}\text { Planned } \\
2019-20 \\
\end{array}$ & $\begin{array}{l}\text { Planned } \\
2020-21\end{array}$ & $\begin{array}{c}\text { Planned } \\
\text { Annual } \\
\text { Growth } \\
\text { Rate }^{*}\end{array}$ \\
\hline \multicolumn{10}{|c|}{ STATE FUNDABLE } \\
\hline \multicolumn{10}{|c|}{ Florida Resident } \\
\hline LOWER & 7,565 & $\mathrm{n} / \mathrm{a}$ & 7,522 & 7,538 & 7,554 & 7,570 & 7,586 & 7,602 & $0.2 \%$ \\
\hline UPPER & 11,468 & $\mathrm{n} / \mathrm{a}$ & 11,348 & 11,372 & 11,396 & 11,420 & 11,445 & 11,469 & $0.2 \%$ \\
\hline GRAD I & 2,973 & $\mathrm{n} / \mathrm{a}$ & 2,886 & 2,938 & 2,991 & 3,045 & 3,100 & 3,156 & $1.8 \%$ \\
\hline GRAD ॥ & 917 & $\mathrm{n} / \mathrm{a}$ & 842 & 857 & 872 & 888 & 904 & 920 & $1.8 \%$ \\
\hline TOTAL & 22,923 & $\mathrm{n} / \mathrm{a}$ & 22,598 & 22,705 & 22,813 & 22,923 & 23,034 & 23,147 & $0.5 \%$ \\
\hline \multicolumn{10}{|l|}{ Non-Resident } \\
\hline LOWER & 836 & $\mathrm{n} / \mathrm{a}$ & 835 & 836 & 838 & 840 & 842 & 844 & $0.2 \%$ \\
\hline UPPER & 803 & $\mathrm{n} / \mathrm{a}$ & 800 & 802 & 804 & 805 & 807 & 809 & $0.2 \%$ \\
\hline GRAD I & 903 & $\mathrm{n} / \mathrm{a}$ & 1,082 & 1,102 & 1,122 & 1,142 & 1,162 & 1,183 & $1.8 \%$ \\
\hline GRAD II & 659 & $\mathrm{n} / \mathrm{a}$ & 669 & 681 & 694 & 706 & 719 & 732 & $1.8 \%$ \\
\hline TOTAL & 3,200 & $\mathrm{n} / \mathrm{a}$ & 3,387 & 3,422 & 3,457 & 3,493 & 3,530 & 3,568 & $1.0 \%$ \\
\hline \multicolumn{10}{|l|}{ TOTAL } \\
\hline LOWER & 8,401 & 9,004 & 8,356 & 8,374 & 8,392 & 8,410 & 8,428 & 8,446 & $0.2 \%$ \\
\hline UPPER & 12,271 & 10,883 & 12,148 & 12,174 & 12,200 & 12,226 & 12,252 & 12,278 & $0.2 \%$ \\
\hline GRAD I & 3,876 & 3,082 & 3,969 & 4,040 & 4,113 & 4,187 & 4,262 & 4,339 & $1.8 \%$ \\
\hline GRAD II & 1,576 & 842 & 1,511 & 1,538 & 1,566 & 1,594 & 1,623 & 1,652 & $1.8 \%$ \\
\hline TOTAL & 26,124 & 23,811 & 25,984 & 26,127 & 26,271 & 26,417 & 26,565 & 26,715 & $0.6 \%$ \\
\hline \multicolumn{10}{|c|}{ NOT STATE FUNDABLE } \\
\hline LOWER & 429 & $\mathrm{n} / \mathrm{a}$ & 441 & 442 & 443 & 444 & 445 & 446 & $0.2 \%$ \\
\hline UPPER & 288 & $\mathrm{n} / \mathrm{a}$ & 264 & 265 & 265 & 266 & 266 & 267 & $0.2 \%$ \\
\hline GRAD I & 470 & $\mathrm{n} / \mathrm{a}$ & 475 & 484 & 493 & 501 & 510 & 520 & $1.8 \%$ \\
\hline GRAD ॥ & 17 & $\mathrm{n} / \mathrm{a}$ & 17 & 17 & 18 & 18 & 18 & 19 & $1.8 \%$ \\
\hline TOTAL & 1,204 & $\mathrm{n} / \mathrm{a}$ & 1,197 & 1,208 & 1,218 & 1,229 & 1,240 & 1,251 & $0.9 \%$ \\
\hline
\end{tabular}

Note: Full-time Equivalent (FTE) student is a measure of instructional effort (and student activity) that is based on the number of credit hours that students enroll. FTE is based on the Florida definition, which divides undergraduate credit hours by 40 and graduate credit hours by 32 . Note*:The average annual growth rate is based on the annual growth rate from 2015-16 to 2020-21.

Medical Student Headcount Enrollments( E\&G funded)

\section{Medical Doctorate Headcounts}

\begin{tabular}{lrcccccccc}
\hline RESIDENT & 438 & 480 & 438 & 421 & 414 & 420 & 420 & 420 & $0 \%$ \\
\hline NON-RESIDENT & 53 & 0 & 49 & 60 & 67 & 60 & 60 & 60 & $0 \%$ \\
\hline TOTAL & 491 & $\mathbf{4 8 0}$ & $\mathbf{4 8 7}$ & $\mathbf{4 8 1}$ & $\mathbf{4 8 1}$ & $\mathbf{4 8 0}$ & $\mathbf{4 8 0}$ & $\mathbf{4 8 0}$ & $\mathbf{0} \%$
\end{tabular}




\section{ACADEMIC PROGRAM COORDINATION}

\section{New Programs For Consideration by University in AY 2015-16}

The S.U.S. Council of Academic Vice Presidents (CAVP) Academic Program Coordination Work Group will review these programs as part of their on-going coordination efforts. The programs listed below are based on the 2014-15 Work Plan list for programs under consideration for 2015-16.

\begin{tabular}{|c|c|c|c|c|c|c|}
\hline PROGRAM TITLES & $\begin{array}{c}\text { CIP CODE } \\
\text { 6-digit }\end{array}$ & $\begin{array}{l}\text { AREA OF } \\
\text { STRATEGIC } \\
\text { EMPHASIS }\end{array}$ & $\begin{array}{c}\text { OTHER } \\
\text { UNIVERSITIES } \\
\text { WITH SAME } \\
\text { PROGRAM } \\
\end{array}$ & $\begin{array}{c}\text { OFFERED VIA } \\
\text { DISTANCE } \\
\text { LEARNING } \\
\text { IN SYSTEM }\end{array}$ & $\begin{array}{l}\text { PROJECTED } \\
\text { ENROLLMENT } \\
\text { in 5th year }\end{array}$ & $\begin{array}{l}\text { PROPOSED } \\
\text { DATE OF } \\
\text { SUBMISSION } \\
\text { TO UBOT }\end{array}$ \\
\hline \multicolumn{7}{|l|}{ BACHELOR'S PROGRAMS } \\
\hline BS Econometrics & 45.0603 & & & $10 \%$ & 60 & Fall 2015 \\
\hline
\end{tabular}

MASTER'S, SPECIALIST AND OTHER ADVANCED MASTER'S PROGRAMS

$\begin{array}{ccccccc}\text { MS Pharmacy } & 51.2099 & \text { HEALTH } & \text { FAMU, UF } & 80 \% & 100 & \text { Fall } 2015 \\ \text { MS Nurse Anesthetist } & 51.3804 & \text { HEALTH } & \text { FIU } & 0 \% & 35 & \text { Fall } 2015\end{array}$

DOCTORAL PROGRAMS

Ph.D. Pharmacy

$51.2099 \quad$ STEM

FAMU, UF

$30 \%$

Spr 2016

New Programs For Consideration by University in 2016-18

These programs will be used in the 2016 Work Plan list for programs under consideration for 2016-17.

\begin{tabular}{ccccccc}
\multicolumn{1}{c}{ PROGRAM TITLES } & $\begin{array}{c}\text { CIP CODE } \\
\text { 6-digit }\end{array}$ & $\begin{array}{c}\text { AREA OF } \\
\text { STRATEGIC } \\
\text { EMPHASIS }\end{array}$ & $\begin{array}{c}\text { OTHER } \\
\text { UNIVRSITIES } \\
\text { WITH SAME } \\
\text { PROGRAM }\end{array}$ & $\begin{array}{c}\text { OFFERED VIA } \\
\text { DISTANCE } \\
\text { LEARNING } \\
\text { IN SYSTEM }\end{array}$ & $\begin{array}{c}\text { PROJECTED } \\
\text { ENROLLMENT } \\
\text { in 5th year }\end{array}$ & $\begin{array}{c}\text { PROPOSED } \\
\text { DATE OF } \\
\text { SUBMISSION } \\
\text { TO UBOT }\end{array}$ \\
\hline BACHELOR'S PROGRAMS & & & & & & \\
\hline BS Public Relations, Adv., Com & 09.0900 & GAP & $\begin{array}{c}\text { FSU, FAMU, } \\
\text { FIU }\end{array}$ & $25 \%$ & 580 & Fall 2016 \\
\hline
\end{tabular}

MASTER'S, SPECIALIST AND OTHER ADVANCED MASTER'S PROGRAMS

\section{DOCTORAL PROGRAMS}

\begin{tabular}{ccccccc} 
Ph.D. Applied Linguistics & 16.0105 & - & - & $0 \%$ & 25 & Fall 2016 \\
\hline OTD Occupational Therapy & 51.2306 & HEALTH & FAMU, FIU & $0 \%$ & 80 & Fall 2017 \\
\hline Ph.D. Behavioral and Com Sci & 44.0000 & - & - & $0 \%$ & 20 & Fall 2017
\end{tabular}




\section{Percent of Bachelor's Graduates Employed Full- time or Continuing their Education in the U.S. One Year After Graduation}

This metric is based on the percentage of a graduating class of bachelor's degree recipients who are employed full-time or continuing their education somewhere in the United States. Students who do not have valid social security numbers and are not found enrolled are excluded. Note: This data now non-Florida employment data.

Sources: State University Database System (SUDS), Florida Education \& Training Placement Information Program (FETPIP) analysis of Wage Record Interchange System (WRIS2) and Federal Employment Data Exchange (FEDES), and National Student Clearinghouse (NSC).

\section{Median Wages of Bachelor's Graduates Employed Full-time in Florida One Year After Graduation}

This metric is based on annualized Unemployment Insurance (UI) wage data from the fourth fiscal quarter after graduation for bachelor's recipients. UI wage data does not include individuals who are self-employed, employed out of state, employed by the military or federal government, those without a valid social security number, or making less than minimum wage.

Sources: State University Database System (SUDS), Florida Education \& Training Placement Information Program (FETPIP), National Student Clearinghouse.

\section{Average Cost per Bachelor's Degree} Instructional costs to the university
For each of the last four years of data, the annual total undergraduate instructional expenditures were divided by the total fundable student credit hours to create a cost per credit hour for each year. This cost per credit hour was then multiplied by 30 credit hours to derive an average annual cost. The average annual cost for each of the four years was summed to provide an average cost per degree for a baccalaureate degree that requires 120 credit hours.

Sources: State University Database System (SUDS), Expenditure Analysis: Report IV.

\section{Six Year FTIC Graduation Rate}

This metric is based on the percentage of first-time-in-college (FTIC) students who started in the Fall (or summer continuing to Fall) term and had graduated from the same institution within six years. Students of degree programs longer than four years (eg, PharmD) are included in the cohorts. Students who are active duty military are not included in the data. Source: State University Database System (SUDS).

\section{Academic}

Progress Rate

2nd Year Retention with GPA Above 2.0
This metric is based on the percentage of first-ime-in-college (FTIC) students who started in the Fall (or summer continuing to Fall) term and were enrolled full-time in their first semester and were still enrolled in the same institution during the Fall term following their first year with had a grade point average (GPA) of at least 2.0 at the end of their first year (Fall, Spring, Summer).

Source: State University Database System (SUDS).

\section{University Access Rate \\ Percent of Undergraduates with a Pell-grant \\ Bachelor's \\ Degrees Awarded within Programs of Strategic Emphasis (includes STEM)}

\section{Graduate \\ Degrees Awarded within Programs of Strategic Emphasis (includes STEM)}

This metric is based the number of undergraduates, enrolled during the fall term, who received a Pellgrant during the fall term. Unclassified students, who are not eligible for Pell-grants, were excluded from this metric. Source: State University Database System (SUDS).

This metric is based on the number of baccalaureate degrees awarded within the programs designated by the Board of Governors as 'Programs of Strategic Emphasis'. A student who has multiple majors in the subset of targeted Classification of Instruction Program codes will be counted twice (i.e., double-majors are included). Source: State University Database System (SUDS).

This metric is based on the number of graduate degrees awarded within the programs designated by the Board of Governors as 'Programs of Strategic Emphasis'. A student who has multiple majors in the subset of targeted Classification of Instruction Program codes will be counted twice (i.e., doublemajors are included). Source: State University Database System (SUDS). 
Freshmen in Top 10\% of High Percent of all degree-seeking, first-time, first-year (freshman) students who had high school class School Class rank within the top $10 \%$ of their graduating high school class.

Applies to: NCF

Source: New College of Florida.

\section{BOG Choice Metrics}

\section{Percent of Bachelor's Degrees Without Excess Hours}

\section{Number of Faculty Awards}

This metric is based on the percentage of baccalaureate degrees awarded within $110 \%$ of the credit hours required for a degree based on the Board of Governors Academic Program Inventory. Note: It is important to note that the statutory provisions of the "Excess Hour Surcharge" (1009.286, FS) have been modified several times by the Florida Legislature, resulting in a phased-in approach that has created three different cohorts of students with different requirements. The performance funding metric data is based on the latest statutory requirements that mandates $110 \%$ of required hours as the threshold. In accordance with statute, this metric excludes the following types of student credits (ie, accelerated mechanisms, remedial coursework, non-native credit hours that are not used toward the degree, non-native credit hours from failed, incomplete, withdrawn, or repeated courses, credit hours from internship programs, credit hours up to 10 foreign language credit hours, and credit hours earned in military science courses that are part of the Reserve Officers' Training Corps (ROTC) program).

Source: State University Database System (SUDS).

This metric is based on the number of awards that faculty have earned in the arts, humanities, science, engineering and health fields as reported in the annual 'Top American Research Universities' report. Twenty-three of the most prominent awards are considered, including: Getty Scholars in Residence, Guggenheim Fellows, Howard Hughes Medical Institute Investigators, MacArthur Foundation Fellows, National Endowment for the Humanities (NEH) Fellows, National Medal of Science and National Medal of Technology, Robert Wood Johnson Policy Fellows, Sloan Research Fellows, Woodrow Wilson Fellows, to name a few awards.

Source: Center for Measuring University Performance, Annual Report of the Top American Research Universities (TARU).

This metric is based on the number of Top 50 university rankings that NCF earned from the following list of publications: Princeton Review, Fiske Guide, QS World University Ranking, Times Higher Education World University Ranking, Academic Ranking of World University, US News and World Report National University, US News and World Report National Public University, US News and World Report Liberal Arts Colleges, Forbes, Kiplinger, Washington Monthly Liberal Arts Colleges, Washington Monthly National University, and Center for Measuring University Performance. Source: Board of Governors staff review.

\section{BOT Choice Metrics}

Percent of R\&D Expenditures Funded from External

Sources

FAMU

Bachelor's Degrees Awarded to Minorities

FAU, FGCU, FIU
This metric reports the amount of research expenditures that was funded from federal, private industry and other (non-state and non-institutional) sources.

Source: National Science Foundation annual survey of Higher Education Research and Development (HERD).

This metric is the number, or percentage, of baccalaureate degrees granted in an academic year to Non-Hispanic Black and Hispanic students. This metric does not include students classified as NonResident Alien or students with a missing race code.

Source: State University Database System (SUDS).

This metric is based on the difference between the Financial Resources rank and the overall University rank. U.S. News measures financial resources by using a two-year average spending per student on instruction, research, student services and related educational expenditures - spending on sports, dorms and hospitals doesn't count.

Source: US News and World Report's annual National University rankings. 


\section{Percent of Undergraduate Seniors Participating in a Research Course NCF}

Number of Bachelor Degrees
Awarded Annually
UCF

Total Research Expenditures UF

Percent of Course Sections
Offered via Distance and
Blended Learning
UNF

This metric is based on the percentage of undergraduate seniors who participate in a research course during their senior year.

Source: New College of Florida.
This metric is the number of baccalaureate degrees granted in an academic year. Students who earned two distinct degrees in the same academic year were counted twice; students who completed multiple majors or tracks were only counted once.

Source: State University Database System (SUDS).

This metric is the total expenditures (includes non-science \& engineering fields) for research \& development activities within a given fiscal year.

Source: National Science Foundation annual survey of Higher Education Research and Development (HERD).

This metric is based on the percentage of course sections classified as having at least $50 \%$ of the instruction delivered using some form of technology, when the student and instructor are separated by time or space, or both. Source: State University Database System (SUDS).

This metric is based on the number of post-doctoral appointees at the beginning of the academic year. A postdoctoral researcher has recently earned a doctoral (or foreign equivalent) degree and has a temporary paid appointment to focus on specialized research/scholarship under the supervision of a senior scholar.

Source: National Science Foundation/National Institutes of Health annual Survey of Graduate Students and Postdoctorates in Science and Engineering (GSS).

\section{Percentage of Adult Undergraduates Enrolled UWF}

This metric is based on the percentage of undergraduates (enrolled during the fall term) who are at least 25 years old at the time of enrollment. This includes undergraduates who are not degreeseeking, or unclassified.

Source: State University Database System (SUDS).

\section{Preeminent Research University Funding Metrics}

Average GPA and SAT Score

Public University National Ranking

\author{
Freshman Retention Rate \\ (Full-time, FTIC)
}

An average weighted grade point average of 4.0 or higher and an average SAT score of 1800 or higher for fall semester incoming freshmen, as reported annually in the admissions data that universities submit to the Board of Governors. This data includes registered FTIC (student type='B','E') with an admission action of admitted or provisionally admitted ('A','P','X').

A top-50 ranking on at least two well-known and highly respected national public university rankings, reflecting national preeminence, using most recent rankings, includes: Princeton Review, Fiske Guide, QS World University Ranking, Times Higher Education World University Ranking, Academic Ranking of World University, US News and World Report National University, US News and World Report National Public University, US News and World Report Liberal Arts Colleges, Forbes, Kiplinger, Washington Monthly Liberal Arts Colleges, Washington Monthly National University, and Center for Measuring University Performance.

Freshman Retention Rate (Full-time, FTIC) as reported annually to the Integrated Postsecondary Education Data System (IPEDS). The retention rates that are reported in the Board's annual Accountability report are preliminary because they are based on student enrollment in their second fall term as reported by the 28th calendar day following the first day of class. When the Board of Governors reports final retention rates to IPEDS in the Spring (usually the first week of April), that data is based on the student enrollment data as reported after the Fall semester has been completed. The preliminary and final retention rates are nearly identical when rounded to the nearest whole number. 
6-year Graduation Rate (Full-time, FTIC)

Cohorts are based on undergraduate students who enter the institution in the Fall term (or Summer term and continue into the Fall term). Percent Graduated is based on federal rate and does not include students who originally enroll as part-time students, or who transfer into the institution. This metric complies with the requirements of the federal Student Right to Know Act that requires institutions to report the completion status at $150 \%$ of normal time (or six years). For more information about how this data is calculated, see:

http://www.flbog.edu/about/budget/docs/performance_funding/PBF_GRADUATION_and_RETENTION_Method ology FINAL.pdf.

National Academy Memberships held by faculty as reported by the Center for Measuring University Performance in the Top American Research Universities (TARU) annual report.

National Academy Memberships

Total Annual Research Expenditures (\$M)

(Science \& Engineering only)
Total Science \& Engineering Research Expenditures, including federal research expenditures, of $\$ 200$ million or more, as reported annually by the National Science Foundation (NSF).
Total Annual Research Expenditures in Diversified NonMedical Sciences (\$M)

(Science \& Engineering only)

National Ranking in S.T.E.M. Research Expenditures

\section{Patents Awarded}

(over 3 year period)
Total S\&E research expenditures in non-medical sciences as reported by the NSF. This removes medical sciences funds ( $9 F \& 12 F$ in HERD survey) from the total S\&E amount.

The NSF identifies 8 broad disciplines within Science \& Engineering (Computer Science, Engineering, Environmental Science, Life Science, Mathematical Sciences, Physical Sciences, Psychology, Social Sciences). The rankings by discipline are determined by BOG staff using the NSF WebCaspar database.

Total patents awarded by the United States Patent and Trademark Office (USPTO) for the most recent 3-year period. Due to a year-lag in published reports, Board of Governors staff query the USPTO database with a query that only counts utility patents:"(AN/"University Name" AND ISD/yyyymmdd->yyyymmdd AND APT/1)".

Doctoral Degrees Awarded Annually

Doctoral degrees awarded annually, as reported annually in the Board of Governors Accountability Report. Note: per legislative workpapers, this metric does not include Professional degrees.

\section{Number of Post-Doctoral Appointees}

Endowment Size (\$M)
The number of Postdoctoral Appointees awarded annually, as reported in the TARU annual report. This data is based on National Science Foundation/National Institutes of Health annual Survey of Graduate Students and Postdoctorates in Science and Engineering (GSS).

This data comes from the National Association of College and University Business Officers (NACUBO) and Commonfund Institute's annual report of Market Value of Endowment Assets - which, due to timing, may release the next fiscal year's data after the Board of Governors Accountability report is published. 


\section{Goals Common to All Universities}

\section{Academic Quality}

Avg. SAT Score (for 3 subtests)

An average weighted grade point average of 4.0 or higher and an average SAT score of 1800 or higher for fall semester incoming freshmen, as reported annually in the admissions data that universities submit to the Board of Governors. This data includes registered FTIC (student type='B','E') with an admission action of admitted or provisionally admitted ('A','P','X').

Avg. HS GPA The average HS GPA for Admitted \& Registered FTIC and early admit (B,E) students. Max score is 5.0 .

Professional/Licensure Exam First-time Pass Rates

The number of exams with first-time pass rates above and below the national or state average, as reported in the annual Accountability report, including: Nursing, Law, Medicine (3 subtests), Veterinary, Pharmacy, Dental (2 subtests), Physical Therapy, and Occupational Therapy.

\section{Operational Efficiency}

Freshman Retention Rate

\section{FTIC Graduation Rates}

In 4 years (or less)

In 6 years (or less)

\section{AA Transfer Graduation Rates} In 2 years (or less)

\section{Average Time to Degree (for FTIC)}

\section{Return on Investment}

Bachelor's Degrees Awarded

Percent of Bachelor's Degrees in STEM

Graduate Degrees Awarded

Percent of Graduate Degrees in STEM

Annual Gifts Received (\$M)

Endowment (\$M)
The percentage of a full-time, first-time-in-college (FTIC) undergraduate cohort (entering in fall term or summer continuing to fall) that is still enrolled or has graduated from the same institution in the following fall term as reported in the annual Accountability report (table 4B) - see link.

As reported in the annual Accountability report (table 4D), First-time-in-college (FTIC) cohort is defined as undergraduates entering in fall term (or summer continuing to fall) with fewer than 12 hours earned since high school graduation. The rate is the percentage of the initial cohort that has either graduated from or is still enrolled in the same institution by the fourth or sixth academic year. Both full-time and part-time students are used in the calculation. The initial cohort is revised to remove students, who have allowable exclusions as defined by IPEDS, from the cohort.

As reported in the annual Accountability report (table 4E), AA Transfer cohort is defined as undergraduates entering in the fall term (or summer continuing to fall) and having earned an AA degree from an institution in the Florida College System. The rate is the percentage of the initial cohort that has either graduated from or is still enrolled in the same institution by the second or fourth academic year. Both full-time and part-time students are used in the calculation. The initial cohort is revised to remove students, who have allowable exclusions as defined by IPEDS, from the cohort. This metric is the number of years between the start date (using date of most recent admission) and the end date (using the last month in the term degree was granted) for a graduating class of first-time, single-major baccalaureates in 120 credit hour programs within a (Summer, Fall, Spring) year.

This is a count of baccalaureate degrees awarded as reported in the annual Accountability Report (table 4G).

The percentage of baccalaureate degrees that are classified as STEM by the Board of Governors in the SUS program inventory as reported in the annual Accountability Report (table $4 \mathrm{H}$ ).

This is a count of graduate degrees awarded as reported in the Accountability Report (table 5B).

in The percentage of baccalaureate degrees that are classified as STEM by the Board of Governors in the SUS program inventory as reported in the annual Accountability Report (table 5C).

As reported in the Council for Aid to Education's Voluntary Support of Education (VSE) survey in the section entitled "Gift Income Summary," this is the sum of the present value of all gifts (including outright and deferred gifts) received for any purpose and from all sources during the fiscal year, excluding pledges and bequests. (There's a deferred gift calculator at www.cae.org/vse.) The present value of non-cash gifts is defined as the tax deduction to the donor as allowed by the IRS.

Endowment value at the end of the fiscal year, as reported in the annual NACUBO Endowment Study (changed to the NACUBO-Common Fund Study of Endowments in 2009). 


\section{Goals Specific to Research Universities}

Academic Quality

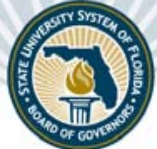

Faculty Awards

\section{National Academy Members}

\section{Number of Post-Doctoral} appointees

\section{Number of Science \& Engineering Disciplines nationally ranked in Top 100 for research expenditures}

Return on Investment Total Research Expenditures (\$M)

Science \& Engineering
Research Expenditures in non-
medical/health
sciences

\section{Percent of R\&D Expenditures funded from External Sources}

\section{Patents Issued}

Licenses/Options Executed

Licensing Income Received (\$M)

Number of Start-up Companies

Awards include: American Council of Learned Societies (ACLS) Fellows, Beckman Young Investigators, Burroughs Wellcome Fund Career Awards, Cottrell Scholars, Fulbright American Scholars, Getty Scholars in Residence, Guggenheim Fellows, Howard Hughes Medical Institute Investigators, Lasker Medical Research Awards, MacArthur Foundation Fellows, Andrew W. Mellon Foundation Distinguished Achievement Awards, National Endowment for the Humanities (NEH) Fellows, National Humanities Center Fellows, National Institutes of Health (NIH) MERIT, National Medal of Science and National Medal of Technology, NSF CAREER awards (excluding those who are also PECASE winners), Newberry Library Long-term Fellows, Pew Scholars in Biomedicine, Presidential Early Career Awards for Scientists and Engineers (PECASE), Robert Wood Johnson Policy Fellows, Searle Scholars, Sloan Research Fellows, Woodrow Wilson Fellows. As reported by the Top American Research Universities - see: http://mup.asu.edu/research_data.html.

The number of National Academy members included in the National Academy of Sciences, National Academy of Engineering, and the Institute of Medicine. As reported by the Top American Research Universities - see: http://mup.asu.edu/research_data.html.

As submitted to the National Science Foundation Survey of Graduate Students and Postdoctorates in Science \& Engineering (also known as the GSS) - see link.

The number of Science \& Engineering disciplines the university ranks in the top 100 (for public and private universities) based on the National Science Foundation's annual survey for R\&D expenditures, which identifies 8 broad disciplines within Science \& Engineering (Computer Science, Engineering, Environmental Science, Life Science, Mathematical Sciences, Physical Sciences, Psychology, and Social Sciences). Historically NSF provided these rankings (see tables 45-61 at link), but now data must be queried via WebCASPAR - see link.

Total expenditures for all research activities (including non-science and engineering activities) as reported in the National Science Foundation annual survey of Higher Education Research and Development (HERD).

This metric reports the Science \& Engineering total R\&D expenditures minus the research expenditures for medical sciences as reported by the National Science Foundation. Historically NSF provided these data (see link, table 36 minus table 52), but now data must be queried via WebCASPAR.

This metric reports the amount of research expenditures that was funded from federal, private industry and other (non-state and non-institutional) sources.

Source: National Science Foundation annual survey of Higher Education Research and Development (HERD).

The number of patents issued in the fiscal year as reported in the annual Accountability Report (table $6 \mathrm{~A})$.

Licenses/options executed in the fiscal year for all technologies as reported in the annual Accountability Report (table 6A).

License issue fees, payments under options, annual minimums, running royalties, termination payments, amount of equity received when cashed-in, and software and biological material end-user license fees of $\$ 1,000$ or more, but not research funding, patent expense reimbursement, valuation of equity not cashed-in, software and biological material end-user license fees of less than $\$ 1,000$, or trademark licensing royalties from university insignia. Data as reported in the annual Accountability Report (table 6A).

The number of start-up companies that were dependent upon the licensing of University technology for initiation as reported in the annual Accountability Report (table 6A).

This metric compares the overall national university ranking to the financial resources rank as reported by the US News and World report.

Ranking based on US News \& World Report

Research Doctoral Degrees Awarded

The number of research doctoral degrees awarded annually as reported in the annual Accountability Report (table 5B). 
Professional Doctoral Degrees Awarded
The number of professional doctoral degrees awarded annually as reported in the annual Accountability Report (table 5B).

\section{Student Debt Summary}

Percent of Bachelor's Recipients with Debt
This is the percentage of bachelor's graduates in a given academic year who entered the university as a first-time-in-college (FTIC) student and who borrowed through any loan programs (institutional, state, Federal Perkins, Federal Stafford Subsidized and unsubsidized, private) that were certified by your institution - excludes parent loans.

Source: Common Dataset $(\mathrm{H} 4)$.

This is the average amount of cumulative principal borrowed (from any loan program certified by the institution) for each native, FTIC bachelor's recipient in a given academic year that graduated with debt - see metric definition above. This average does NOT include students who did not enter a loan program that was certified by the institution.

Source: Common Dataset (H5).

Student loan cohort default rate (CDR) data includes undergraduate and graduate students, and refers to the three federal fiscal year period when the borrower enters repayment and ends on the second fiscal year following the fiscal year in which the borrower entered repayment. Cohort default rates are based on the number of borrowers who enter repayment, not the number and type of loans that enter repayment. A borrower with multiple loans from the same school whose loans enter repayment during the same cohort fiscal year will be included in the formula only once for that cohort fiscal year. Default rate debt includes: Federal Stafford Loans, and Direct Stafford/Ford Loans - for more information see: http://ifap.ed.gov/DefaultManagement/CDRGuideMaster.html.

\begin{tabular}{|c|c|c|c|}
\hline \multicolumn{4}{|c|}{ Three Year CDR } \\
\hline $\begin{array}{l}\text { Cohort } \\
\text { Fiscal } \\
\text { Year }\end{array}$ & $\begin{array}{c}\text { Year } \\
\text { Published }\end{array}$ & $\begin{array}{l}\text { Borrowers in the Numerator } \\
\text { Borrowers in the Denominator }\end{array}$ & $\begin{array}{l}\frac{\text { 3-Yr Time Period }}{\text { (Numerator) }} \\
\text { 1-Yr Time Period } \\
\text { (Denominator) }\end{array}$ \\
\hline 2009 & 2012 & $\begin{array}{l}\text { Borrowers who entered repayment in } 2009 \\
\text { and defaulted in 2009, } 2010 \text { or } 2011 \\
\text { Borrowers who entered repayment in } 2009\end{array}$ & $\frac{10 / 01 / 2008 \text { to } 9 / 30 / 2011}{10 / 01 / 2008 \text { to } 9 / 30 / 2009}$ \\
\hline 2010 & 2013 & $\begin{array}{l}\text { Borrowers who entered repayment in } 2010 \\
\text { and defaulted in } 2010,2011 \text { or } 2012 \\
\text { Borrowers who entered repayment in } 2010\end{array}$ & $\frac{10 / 01 / 2009 \text { to } 9 / 30 / 2012}{10 / 01 / 2009 \text { to } 9 / 30 / 2010}$ \\
\hline 2011 & $2014 *$ & $\begin{array}{l}\text { Borrowers who entered repayment in } 2011 \\
\text { and defaulted in } 2011,2012 \text { or } 2013 \\
\text { Borrowers who entered repayment in } 2011\end{array}$ & $\frac{10 / 01 / 2010 \text { to } 9 / 30 / 2013}{10 / 01 / 2010 \text { to } 9 / 30 / 2011}$ \\
\hline 2012 & 2015 & $\begin{array}{l}\text { Borrowers who entered repayment in } 2012 \\
\text { and defaulted in } 2012,2013 \text { or } 2014 \\
\text { Borrowers who entered repayment in } 2012\end{array}$ & $\frac{10 / 01 / 2011 \text { to } 9 / 30 / 2014}{10 / 01 / 2011 \text { to } 9 / 30 / 2012}$ \\
\hline 2013 & 2016 & $\begin{array}{l}\text { Borrowers who entered repayment in } 2013 \\
\text { and defaulted in } 2013,2014 \text { or } 2015 \\
\text { Borrowers who entered repayment in } 2013\end{array}$ & $\frac{10 / 01 / 2012 \text { to } 9 / 30 / 2015}{10 / 01 / 2012 \text { to } 9 / 30 / 2013}$ \\
\hline 2014 & 2017 & $\begin{array}{l}\text { Borrowers who entered repayment in } 2014 \\
\text { and defaulted in } 2014,2015 \text { or } 2016 \\
\text { Borrowers who entered repayment in } 2014\end{array}$ & $\frac{10 / 01 / 2013 \text { to } 9 / 30 / 2016}{10 / 01 / 2013 \text { to } 9 / 30 / 2014}$ \\
\hline 2015 & 2018 & $\begin{array}{l}\text { Borrowers who entered repayment in } 2015 \\
\text { and defaulted in } 2015,2016 \text { or } 2017 \\
\text { Borrowers who entered repayment in } 2015\end{array}$ & $\frac{10 / 01 / 2014 \text { to } 9 / 30 / 2017}{10 / 01 / 2014 \text { to } 9 / 30 / 2015}$ \\
\hline
\end{tabular}

